\title{
Mathematical modeling of dispersal phenomenon in biofilms
}

\author{
B. D’Acunto ${ }^{\mathrm{a}}$, L. Frunzo ${ }^{\mathrm{a}, *}$, I. Klapper ${ }^{\mathrm{b}}$, M.R. Mattei ${ }^{\mathrm{a}}$, P. Stoodley ${ }^{\mathrm{c}, \mathrm{d}}$ \\ aUniversity of Naples "Federico II", Department of Mathematics and Applications, via Claudio 21, 80125, Naples, Italy \\ ${ }^{b}$ Temple University, Department of Mathematics, 1805 N. Broad St., Philadelphia, 19122, Pennsylvania, USA \\ ${ }^{c}$ Center for Microbial Interface Biology, Departments of Microbial Infection and Immunity and Orthopaedics, The Ohio State University, \\ Columbus, OH 43235. USA \\ ${ }^{d}$ National Centre for Advanced Tribology, Engineering and the Environment, University of Southampton, Southampton, UK
}

\begin{abstract}
A mathematical model for dispersal phenomenon in multispecies biofilm based on a continuum approach and mass conservation principles is presented. The formation of dispersed cells is modeled by considering a mass balance for the bulk liquid and the biofilm. Diffusion of these cells within the biofilm and in the bulk liquid is described using a diffusion-reaction equation. Diffusion supposes a random character of mobility. Notably, biofilm growth is modeled by a hyperbolic partial differential equation while the diffusion process of dispersed cells by a parabolic partial differential equation. The two are mutually connected but governed by different equations that are coupled by two growth rate terms. Three biological processes are discussed. The first is related to experimental observations on starvation induced dispersal [1]. The second considers diffusion of a non-lethal antibiofilm agent which induces dispersal of free cells. The third example considers dispersal induced by a self-produced biocide agent.
\end{abstract}

Keywords: Multispecies biofilms, Biofilm motility, Biofilm dispersal, Nonlinear hyperbolic and parabolic partial differential equations, Numerical simulations, Free boundary problems.

\section{Introduction}

Biofilms are microbial aggregates organized as microcolonies and encased in a matrix of highly hydrated esopolysaccharide substances (EPS). These microbial aggregates can be attached to a living or inert surface (substratum) or constitute suspended cell aggregates defined as floating biofilms (e.g. flocs or granules), which are not attached to a surface but which share the characteristics of a biofilm. Multispecies biofilm can form stable microconsortia, develop physiochemical gradients, and undergo horizontal gene transfer and complex cell-cell communication [2]. This structure might even be considered as an immobilized enzyme system in which the milieu and the enzyme activities are constantly changing and evolving to an approximately steady state. This steady state can then be radically altered by applying physical forces such as high shear, or via external or internal reactions that cause the detachment and loss of regions of the biofilm [3]. Further, they are heterogeneous: several studies show that microbial biofilms are highly hydrated open structures containing a high fraction of exopolymers and large void space and channels [4].

The organization of these complex surface-associated communities enables their microorganisms to develop coordinated and efficient survival strategies. Although the resulting survival ability is useful from the microbial standpoint, the persistence capacity of biofilm can be detrimental. Biofilms may cause chronic infection, parasitism phenomena

\footnotetext{
${ }^{*}$ Corresponding author

Email addresses: dacunto@unina.it (B. D’Acunto), luigi.frunzo@unina.it (L. Frunzo), klapper@temple.edu (I. Klapper), mariarosaria.mattei@unina.it (M.R. Mattei), paul.stoodley@osumc.edu (P. Stoodley)
} 
in animals and plants, biodeterioration of historical heritage objects, and engineering and industrial systems. As a result, biofilm related problems present significant socio-economic costs [5].

In the last two decades, there have been many experimental advances that have clarified the structure, function and genetic expression of biofilms. These experiments have revealed striking commonalities between different species [6]. One such commonality is the biofilm "life cycle" [7] which describes the development of biofilm system. The formation of a biofilm is a complex and dynamic process formed by several steps: surface adhesion of bacteria, formation of an attached monolayer and cell proliferation (microcolonies), development of a mature biofilm, and dispersal as schematically depicted in fig. 1. In contrast to passive dispersal, resulting from sloughing of cell clusters and erosion from the biofilm, the production of specific dispersal cells at the final stage of the biofilm life cycle is an active and highly regulated response [8]. Stress as a result of habitat decay (e.g. resource depletion, cell competition for space) is the most cited explanation for the evolution of the dispersal phase in a biofilm [7]. We note as an aside that a consequence of these observations is that new removal biofilm strategies have been proposed: the idea is not to kill the microrganisms but to induce them to disperse. This approach offers an elegant way of biocide-free biofilm removal [9].

In complement to recent and extensive experimental activity, complex mathematical models and numerical simulations have been proposed to investigate development, structures, and ecological interactions of biofilms [10, 11, 12]. While there is a substantial amount of literature available on detachment models for biofilms, little attention has been directed towards motility and dispersal in biofilms. Only a few papers propose models which include mechanisms for biofilm dispersal: a cellular automaton model for nutrient limited dispersal was presented in [10, 12, 13, 14, 15] and an individual based model for detachment induced by EPS breakdown was introduced in [16]. A partial differential equation based model for cells dispersal was presented in [17], where the authors modeled the dynamics of quorum sensing induced bacterial cell dispersal in growing biofilms. Qualitative analysis of this model has been recently presented in [18]. Here, a mathematical model able to describe dispersal phenomenon as a response to diverse external stress cues, is presented. As examples, the cues triggering the phenomenon such as substrate starvation, presence of toxic substances or an auto-produced biocide agent have been included in the model. Moreover, the model describes the biofilm dynamics under these stress situations through a new and original pathway for biofilm evolution. To this end, a previous motility model is further developed in order to describe the diffusion of planktonic cells originating from dispersal and to highlight the impact they can exert on the ecological functionality of biofilms.

The approach is based on a continuum model in one space dimension [19, 20, 21, 22, 23] with the intention of predicting biofilm growth, spatial distribution of microbial species, substrate trends, attachment and detachment, and in particular biofilm dispersal and the motility of the resulting dispersed cells. The model is based on empirical observations revealing that the biofilm is not a completely impenetrable structure, but rather can be considered as an environment where bacteria can move partially freely. These free bacteria can be responsible for a number of phenomena (e.g. invasion, horizontal gene transfer (HGT), dispersal) relevant to biofilm colony development. Many experimental studies have been devoted to elucidation of the main mechanisms which underly dispersal. In particular, the main causes have been proposed to be related to lack of nutrients or presence of inhibiting substances. In [1], the authors showed that the beginning of the dispersal phase coincides with the formation of necrosis zones, where the active biomass, constituted by active cells, is hydrolyzed leading to the formation of dispersed cells which release in the biofilm and then into the bulk liquid. The release of dispersed cells causes the formation of voids resulting in an increase of biofilm porosity. Mathematically, the dependance of dispersal on substrate dynamics can be taken into account and in particular the phenotypic change from sessile to planktonic can be modeled by considering the inactive biomass as an intermediate particulate component. When dispersal occurs the active biofilm biomass has been modeled to stop its normal metabolic activities and switch to an inactive status. The resulting inactive biomass has been modeled to evolve to inert material or to dispersed cells. Formation of voids has been incorporated in the model by considering a direct dependence of biofilm porosity on the release of free cells. Motility of dispersed cells has been modeled by considering a mass balance for the bulk liquid and the biofilm. Diffusion of dispersed cells within biofilm and in the bulk liquid has been described by using a diffusion-reaction equation, supposing a random character of mobility. The model demonstrates the possibility that the resulting dispersed bacteria can colonize a new area characterized by favorable environmental conditions.

Notably, growth is modeled by a hyperbolic partial differential equation and diffusion of dispersed cells by a parabolic partial differential equation. The two are mutually connected but governed by different equations that are coupled by introducing two growth rate terms. The first, introduced in this work, arises from inactive biomass 

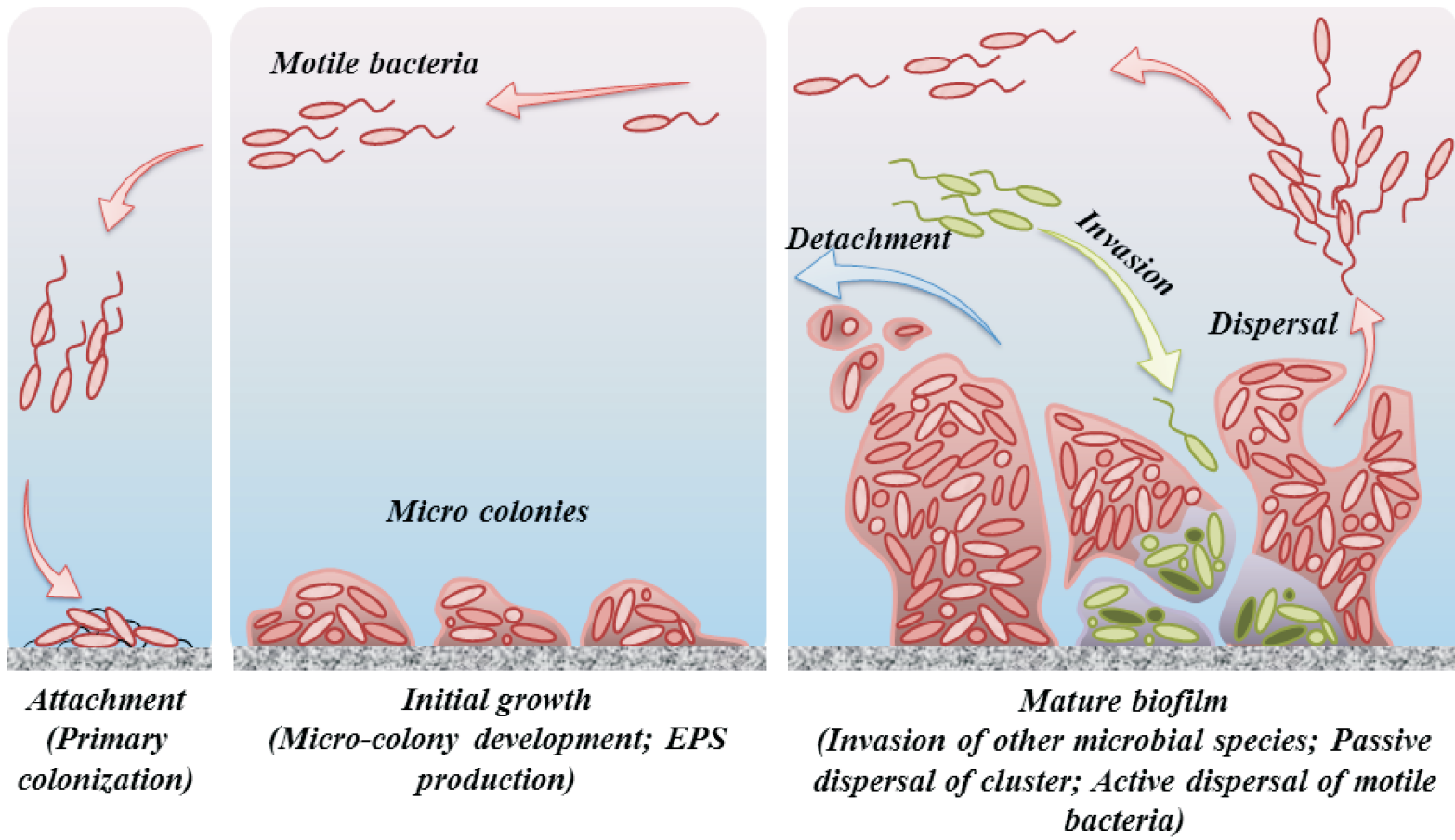

Figure 1. Biofilm life cycle. I) Motile cells colonize a clean surface. II) Development of the single-species microcolonies and EPS production. III) Biofilm colonization of invading microbial species. Loss of biomass (cell clusters) due to mechanical detachment. Loss of biomass (motile cells) due to dispersal.

concentrations in the hyperbolic equations governing biofilm development. This term is connected with parabolic equations governing diffusion processes for dissolved substrates and reproduces the dispersal process. The second takes into account colonization processes [24]. Overall we obtain a hyperbolic free boundary value problem with non-linearities for the biomass distribution and a semi-linear system of second-order boundary value problems for the substrates and the dispersed cells in the biofilm. Note that no boundary conditions on the microbial families are prescribed. This important feature provides generality to the model and can be useful in simulating real situations.

As will be elucidated in Section 2, dispersal can strongly affect the evolution of biofilm in several cases. The aim of the proposed model is to analyze these biological situations not accessible to existing models. In particular, the model is applied to three illustrative cases. The first is based on the experimental observations of Schleheck et al. [1] and traces the dynamics of a Pseudomonas aeruginosa (PA) biofilm which experiences dispersal induced by nutrient starvation. The second involves the presence of a non-lethal antibiofilm agent (i.e. does not affect cell viability but does induce a dispersal response) which interferes with active PA biomass and induces dispersal. The third concerns mathematical modeling of auto-induced dispersal due to the self-production of a biocide. Numerical simulations provide results in reasonable agreement with the experiences and confirm the capability of the model to predict biomass distribution, substrate concentration trends over biofilm depth, release of dispersed cells and their further diffusion within the biofilm to the bulk liquid.

The paper is organized as follows. Section 2 introduces biological details concerning dispersal mechanisms. In Section 3 we present the developed model describing the dispersal phase and its effects on biofilm evolution. In Section 4 some qualitative properties of solutions are proved, mainly to show consistency of the model. Section 5 describes three experimental cases to which the new model is applied and presents the numerical results. Finally, in Section 6 we present conclusions. 


\section{Experimental observations}

Depletion of resources and competition for space are usually considered as the main factors affecting the evolution of the dispersal phase in biofilms. Several theories argue, supported by experimental results $[25,26]$, that many cells in biofilms appear to be in stationary phase. In stationary phase the cells are able to change their status from sessile to motile when adverse conditions occur. Dispersal plays a key role in biofilm development.

It was observed that when biofilm populations are subjected to growth limiting conditions and/or adverse environmental conditions, aggregates may disperse and a significant number of single cells are released (seeding dispersal) [1]. Dispersal is not only a phase of the biofilm life cycle, but also can be an active regulated response when adverse conditions arise. During the last two decades there have been many studies investigating environmental cues that trigger dispersal from biofilm. These cues fundamentally reflect the variation in habitat or environmental stresses, including reduced availability of nutrients (carbon or nitrogen source), oxygen depletion, change in temperature, high or low level of micro nutrients (e.g. heavy metals), and presence of inhibiting agents. Active dispersal from biofilm can be preceded by the formation of necrotic zones within the mature biofilm structure [7]. For some well-studied biofilm systems, it has been possible to determine that cell death within biofilm plays a crucial role in regulating dispersal. Indeed, in Gram negative motile species, dispersal involves the death and lysis of a subpopulation of cells located in the center of the colony with the simultaneous release of highly motile cells (e.g. dispersed cells). The dead cells provide nutrients for the dispersing ones. Further, the release of dispersed cells results in formation of voids and a consequent increase in porosity. Residual biomass shows a slimy and sparse texture, and appears to lack viable cells [1].

Decreases in nutrient concentration have been correlated to biofilm dispersal [27]. For example, work of Schleheck et al. [1] shows the evolution of dispersalin response to both carbon source (glucose) depletion and oxygen depletion for a pseudomonas aeruginosa PAOI (PA) biofilm. More precisely, experimental results show that when starvation occurs, dispersal rapidly ensues and a significant number of dispersed cells are released. In the work of Gjermansen et al. [28], the molecular mechanism triggered by carbon source depletion for a Pseudomonas Putida biofilm is described. They show that nutrient starvation results in a reduction in levels of the adhesin LapA, a major component of biofilm matrix.

Dispersal can also be induced by oxygen starvation (hypoxia or anoxia), an environmental stress that is often experienced by biofilm cells. In many biofilm systems, oxygen represents the least available substrate due to its relatively poor water solubility. It has also been demonstrated that nitric oxide (NO) can play a role in the regulation of dispersal in biofilm. External addition of nontoxic concentrations of NO in the nanomolar range was found to induce dispersal in PA biofilm. Other studies have identified $\mathrm{NO}$ as an important factor in the regulation of biofilm dispersal and survival in Staphylococcus aureus and various single and multispecies biofilm [29]. In particular, low concentrations of NO were shown to induce a transition from the sessile mode of growth to the motile planktonic phenotype [30].

\section{Mathematical model}

\subsection{Model assumptions}

In this section, we develop a mathematical model capable of tracking the dynamics of a mature multispecies biofilm experiencing a dispersal phase, with particular attention to modeling of planktonic cells' release from biofilm matrix and subsequent mobility within the biofilm and in the bulk liquid. The biofilm is assumed to grow in one space direction, denoted $z$, perpendicular to the substratum located at $z=0$. Naturally occurring biofilms consist of microbial communities of different species, cooperating through commensal interactions or competing for space and common resources. Thus we introduce $n$ different particulate components some or all of which could be active microbial species (total number $N \leq n$ ), or other quantities like phenotypic variants (i.e., inactive microbial species), extracellular material, inert biomass, free water, etc., each expressed in terms of concentrations $X_{i}=\rho_{i} f_{i}(z, t)$ or, assuming $\rho_{i}$ constant, equivalently in terms of volume fractions $f_{i}(z, t)$ with $\sum_{i=1}^{n} f_{i}=1$. In particular, according to [21] $\rho_{i}$ is assumed constant in time and in space but with different constants in each biofilm component. The active microbial species consume $m$ dissolved components, named substrates and expressed in terms of concentrations $S_{j}(z, t)$, $j=1, \ldots, m, \mathbf{S}=\left(S_{1}, \ldots, S_{m}\right)$. Substrates are transported by Fickian diffusion from the bulk liquid to the biofilm [21], where they take part in microbial metabolism. Over the course of a biofilm life cycle, active microbial species might 
Biofilm

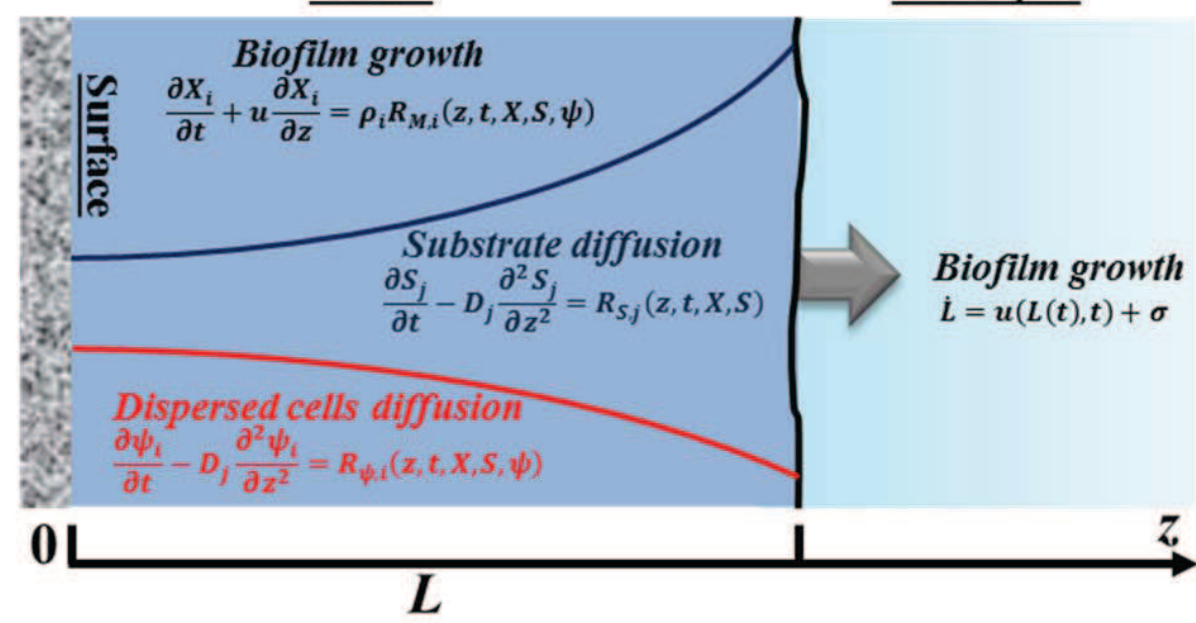

Figure 2. Conceptual representation of the mathematical model. The biofilm growth process is modeled by a system of hyperbolic partial differential equations. Substrates and dispersed cells are modeled by two systems of parabolic partial differential equations.

experience variation in their habitat or environmental quality, including variation in availability of nutrients (carbon or nitrogen source), oxygen depletion, change in temperature, high or low level of micro nutrients (e.g. heavy metals), and the presence of inhibiting agents (e.g. (cis)-2-decenoic acid, trans-bromoageliferin (TAGE), cis-bromoageliferin (CAGE), Oroidin derivates, etc.). Under these conditions, some of the active microbial cells constituting the biofilm are assumed to switch their phenotypic state from sessile to planktonic giving rise to the so called dispersal phenomenon which results in the release of dispersed cells in the aqueous phase, here modeled through Fickian diffusion. The diffusion of dispersed cells from biofilm to bulk liquid and vice versa, has been modelled by using a diffusionreaction equation, following the approach introduced in [24]. This implicates that the volume of dispersed bacteria is zero instantly. This assumption is based on the approximation that the volume occupied by the single planktonic cells is negligible compared to the volume associated to the other biofilm components. Spread through diffusion supposes random mobility. Different characterizations of bacterial movement are possible by considering a diffusion constant dependent on biofilm composition or by using chemotactically-driven mobility. In addition, the release of dispersed cells has two effects: the first is an increase in biofilm porosity due to the loss of cell biomass, the second is an increase in undispersed, inert material due to the loss of viable cells from the biofilm matrix. The increase of porosity balances the loss of volume due to the release of viable cells without affecting the biofilm thickness, with the respect of the conservation of volume. The concentration of dispersed cells is defined by the variables $\psi_{i}(z, t), i=1, . ., N$. Furthermore, detachment represents the only biofilm loss term. Indeed, the framework presented in this work does not allow the biofilm to contract (reduce in thickness) due to dispersal as the conversion of inactive bacteria into dispersed cells is counterbalanced by the increase in biofilm pore fraction.

Altogether, the model is formulated as a system of partial differential equations which can be grouped as follows.

\subsection{Equations for biofilms}

The dynamics of particulate components are governed by the equations

$$
\frac{\partial f_{i}}{\partial t}+\frac{\partial}{\partial z}\left(u f_{i}\right)=R_{M, i}(z, t, \mathbf{f}, \psi, \mathbf{S}), i=1, \ldots, n, 0 \leq z \leq L(t), t>0,
$$


where

$$
R_{M, i}=r_{M, i}(z, t, \mathbf{f}, \mathbf{S})+r_{c o l, i}(z, t, \psi, \mathbf{S}) .
$$

Summing eqs. (1) on $i$ and requiring $\sum_{i=1}^{n} f_{i}=1$ yields

$$
\frac{\partial u}{\partial z}=\sum_{i=1}^{n} R_{M, i}(z, t, \mathbf{f}, \psi, \mathbf{S}), 0<z \leq L(t), t \geq 0 .
$$

The hyperbolic equations (1) describe the evolution, in space and time, of microbial species in the biofilm as a convective flux regulated by the bioconversion of organic substrates and by the phenomena of dispersal and invasion through the term $R_{M, i}$. More precisely: $r_{M, i}(z, t, \mathbf{f}, \mathbf{S})$ is the specific growth rate of a microbial species $i$ due to the bioconversion of substrates, and $r_{c o l, i}(z, t, \psi, \mathbf{S})$ denotes the specific growth rate due to the conversion of bacterial cells from planktonic to sessile status, which characterizes the invasion/colonizing phaenomena. This last term was first introduced by the authors in [24]. Note that equations (1) are used to describe the dynamics of active microbial species as well as all particulate components which constitute the biofilm, including inert biomass, EPS and voids. Note that inert material is surrogated as a fictitious microbial species whose growth derives from the decay and inactivation of active biomass. EPS production is related to biomass metabolism [31], while formation of voids depends on dispersal phenomena. Eq. (1) is written in general form, though of course, for some components (i.e., inactive biomasses, inert, EPS, voids), the term $r_{c o l, i}$ can be zero. Indeed, sorption phenomena, for example passive sequestration of dead cells or inert particles, transported by the flow field and further entrapped into the biofilm matrix are not described by $r_{c o l, i}(z, t, \psi, \mathbf{S})$.

The following initial conditions are considered for Eqs. (1) and (3)

$$
f_{i}(z, 0)=\varphi_{i}(z), i=1, \ldots, n, 0 \leq z \leq L_{0},
$$

where the functions $\varphi_{i}(z), i=1, \ldots, n$, represent the initial volume fractions of microbial species and condition $u(0, t)=$ 0 is a no flux condition between substratum and biofilm.

\subsection{Equations for motility of dispersed cells}

Consider a control volume $\left(z_{2}-z_{1}\right) A$, with $A$ representing biofilm area parallel to the substratum, and denote by $w_{i}(z, t)$ the flux of planktonic species across $A(z)$. The mass balance for species in planktonic state $i=1, \ldots, N$, is

$$
\begin{gathered}
A \frac{\partial}{\partial t} \int_{z_{1}}^{z_{2}} \psi_{i} d z=A\left[w_{i}\left(z_{1}, t\right)-w_{i}\left(z_{2}, t\right)\right]-A \int_{z_{1}}^{z_{2}} r_{c o l, \psi_{i}} d z+A \int_{z_{1}}^{z_{2}} r_{d i s p, \psi_{i}} d z, \\
\int_{z_{1}}^{z_{2}} \frac{\partial \psi_{i}}{\partial t} d z=-\int_{z_{1}}^{z_{2}} \frac{\partial w_{i}}{\partial z} d z-\int_{z_{1}}^{z_{2}} r_{c o l, \psi_{i}} d z+\int_{z_{1}}^{z_{2}} r_{d i s p, \psi_{i}} d z
\end{gathered}
$$

where $r_{d i s p, \psi_{i}}$ represents the production rate of dispersed cells due to dispersal and $r_{c o l \psi, i}$ denotes the planktonic species loss term due to attachment. Note that the term $r_{d i s p, \psi_{i}}$, introduced here depends on both the concentration of microbial species and of substrates within the biofilm and is intended to model the phenotypic change from sessile to planktonic. Equation (5) holds for any control volume $\left(z_{2}-z_{1}\right) A$, therefore the integral functions are equal and the integral can be dropped, leading to the following differential equation

$$
\frac{\partial \psi_{i}}{\partial t}+\frac{\partial}{\partial z}\left(w_{i}\right)=R_{\psi, i}, R_{\psi, i}=r_{d i s p, \psi_{i}}(z, t, \mathbf{X}, \mathbf{S})-r_{c o l \psi, i}(z, t, \psi, \mathbf{S}) .
$$

According to Fick's first law, the flux of colonizing species $i$ within the biofilm is proportional to the diffusivity $D_{M, i}$ and may be expressed as

$$
w_{i}=-D_{M, i} \frac{\partial \psi_{i}}{\partial z}
$$

Substituting eq. (7) into equation (6) leads to

$$
\frac{\partial \psi_{i}}{\partial t}-\frac{\partial}{\partial z}\left(D_{M, i} \frac{\partial \psi_{i}}{\partial z}\right)=R_{\psi, i}(z, t, \psi, \mathbf{X}, \mathbf{S}), i=1, \ldots, n, 0<z<L(t), t>0,
$$


where $D_{M, i}$ denotes the diffusivity coefficient of planktonic species $i . \quad R_{\psi, i}(z, t, \psi, \mathbf{X}, \mathbf{S})$ is the species $i$ conversion rate into the planktonic state and describes the consumption (colonization phenomena) and/or the excretion (dispersal phenomena) of free cells.

Initial-boundary conditions for for Eqs. (8) are prescribed as

$$
\begin{gathered}
\psi_{i}(z, 0)=\psi_{i 0}(z), i=1, \ldots, N, 0 \leq z \leq L(0), \\
\frac{\partial \psi_{i}}{\partial z}(0, t)=0, \quad \psi_{i}(L(t), t)=\psi_{i}^{*}(t), i=1, \ldots, N, t>0 .
\end{gathered}
$$

The functions $\psi_{i 0}(z)$ denote the initial concentrations of planktonic species within the biofilm. Condition (10) $)_{1}$ follows from no flux conditions at the substratum. The second condition in $(10)_{2}$ assigns planktonic species values on the moving boundary $\mathrm{z}=\mathrm{L}(\mathrm{t})$. The functions $\psi_{i}^{*}(t)$ represent the concentrations of planktonic species in the bulk liquid (assumed uniform in space). They can be prescribed or derived from mass balance equations within the bulk liquid. A special form of such mass balance will be introduced in Section 5.

\subsection{Equations for substrates}

Substrate profiles are governed by the equations

$$
\frac{\partial S_{j}}{\partial t}-\frac{\partial}{\partial z}\left(D_{S, j} \frac{\partial S_{j}}{\partial z}\right)=r_{S, j}(z, t, \mathbf{X}, \mathbf{S}), j=1, \ldots, m, 0<z<L(t), t>0,
$$

standard reaction-diffusion equations governing substrate transport from bulk liquid into the biofilm. $r_{S, j}(z, t, \mathbf{X}, \mathbf{S})$ is the conversion rate of substrate $j$ and describes consumption (substrate uptake from microbial biomass occurring during the metabolism process) and/or production (catabolites). $D_{S, j}$ denotes the diffusivity coefficient of substrate $j$.

Initial conditions for substrates are

$$
S_{j}(z, 0)=S_{j 0}(z), 0 \leq z \leq L_{0}, j=1, \ldots, m,
$$

and boundary conditions are

$$
\frac{\partial S_{j}}{\partial z}(0, t)=0, S_{j}(L(t), t)=S_{j}^{*}(t), t>0, j=1, \ldots, m
$$

Conditions $(13)_{1}$ are no flux conditions at the substratum. On the free boundary $z=L(t)$, the functions $S_{j}^{*}(t)$ are the concentrations of the substrates in the bulk liquid, which, similarly to dispersed cells, can be assigned or required to satisfy a mass balance equation in the bulk liquid. A special form of such mass balance will be introduced in Section 5 .

\subsection{Free boundary}

Consider the global mass balance on $[0, L(t)]$ and denote by $\rho_{i} \sigma_{a, i}(t)$ and $\rho_{i} \sigma_{d, i}(L(t))$ respectively the attachment and detachment terms.

$$
\frac{\partial}{\partial t} \int_{0}^{L(t)} \rho_{i} f_{i} d z=\rho_{i} \sigma_{a, i}-\rho_{i} \sigma_{d, i}+\int_{0}^{L(t)} \rho_{i} R_{M, i} d z, i=1, \ldots, n, t>0 .
$$

Summing on $i$ and using (3) and the second equation of (4) yields

$$
\dot{L}(t)=u(L(t), t)+\sigma_{a}(t)-\sigma_{d}(L(t)), t>0, \sigma_{a}(t)=\sum_{i=1}^{n} \sigma_{a, i}, \sigma=\sum_{i=1}^{n} \sigma_{d, i} .
$$

Eq. (15) governs the evolution of the biofilm free boundary, where $L(t)$ denotes biofilm thickness, $\sigma_{a}(t)$ is the attachment biomass flux from bulk liquid to biofilm, and $\sigma_{d}(L(t))$ is the detachment biomass flux from biofilm to bulk liquid. The initial condition for equation (15) is the initial biofilm thickness $L(0)=L_{0}$. 


\subsection{Complete model}

The free boundary value problem is now completely described by the following set of differential equations: the hyperbolic eqs. (1) for the biofilm volume fractions, the non-linear parabolic eqs. (8) for dispersed cells concentrations, the semi-linear parabolic eqs. (11) for substrate concentrations and the two ordinary eqs. for the velocity of the microbial mass (3) and the free boundary evolution (15).

The complete model takes the following form:

$$
\begin{gathered}
\left\{\begin{array}{l}
\frac{\partial f_{i}}{\partial t}+\frac{\partial}{\partial z}\left(u f_{i}\right)=R_{M, i}(z, t, \psi, \mathbf{f}, \mathbf{S}), 0 \leq z \leq L(t), t>0 \\
f_{i}(z, 0)=\varphi_{i}(z), i=1, \ldots, n, 0 \leq z \leq L_{0}
\end{array}\right. \\
\left\{\begin{array}{l}
\frac{\partial u}{\partial z}=\sum_{i=1}^{n} R_{M, i}(z, t, \mathbf{f}, \mathbf{S}), 0<z \leq L(t), t \geq 0 \\
u(0, t)=0, t>0,
\end{array}\right. \\
\left\{\begin{array}{l}
\dot{L}(t)=u(L(t), t)+\sigma_{a}(t)-\sigma_{d}(L(t)), t>0 \\
L(0)=L_{0}
\end{array}\right. \\
\left\{\begin{array}{c}
\frac{\partial \psi_{i}}{\partial t}-\frac{\partial}{\partial z}\left(D_{M, i} \frac{\partial \psi_{i}}{\partial z}\right)=R_{\psi, i}(z, t, \boldsymbol{\psi}, \mathbf{X}, \mathbf{S}), i=1, \ldots, N, 0<z<L(t), t>0, \\
\psi_{i}(z, 0)=0,0 \leq z \leq L_{0}, \frac{\partial \psi_{i}}{\partial z}(0, t)=0, \psi_{i}(L(t), t)=\psi_{i}^{*}(t), \\
i=1, \ldots, N, t>0,
\end{array}\right. \\
\left\{\begin{array}{c}
\frac{\partial S_{j}}{\partial t}-\frac{\partial}{\partial z}\left(D_{S, j} \frac{\partial S_{j}}{\partial z}\right)=r_{S, j}(z, t, \mathbf{X}, \mathbf{S}), j=1, \ldots, m, 0<z<L(t), t>0 \\
S_{j}(z, 0)=S_{j 0}(z), 0 \leq z \leq L_{0}, \frac{\partial S_{j}}{\partial z}(0, t)=0, S_{j}(L(t), t)=S_{j}^{*}(t), \\
j=1, \ldots, m, t>0
\end{array}\right.
\end{gathered}
$$

The hyperbolic equations are used to model the active microbial species (active biomasses) dynamics as well as other components constituting a mature biofilm (e.g. inert material, EPS). Other components are introduced in the form of the inactive microbial species or inactive biomasses. The latter can be considered as intermediate states between active and inert biomasses (fig.3) and are preparatory to dispersal (see Section 2). During the dispersal phase, inactive biomass becomes inert with the simultaneous release of dispersed cells reproducing the necrotic zones experimentally observed in [1] (see 2).

We specify indices in system (16)-(20) as follows. Designate the active microbial species by the indexes $i=$ $1, . ., N$; the inactive microbial species by the indexes $i=N+1, . ., 2 N$; inert materials by the index $i=2 N+1$; EPS by the index $i=2 N+2$ and the liquid content by the index $i=2 N+3$.

For the specific studies presented here, the kinetic terms $r_{M i}(z, t, \mathbf{X}, \mathbf{S})$ for the active microbial species can be expressed as follows:

$$
r_{M, i}=\left(\left(1-K_{E P S, i}\right) \mu_{i}-k_{d, i}-\mu_{I, i}\right) f_{i}, i=1, \ldots, N,
$$

The term $\left(1-K_{E P S, i}\right) \mu_{i}$ represents the specific growth rate of the active biomass due to the uptake of substrates. $\mu_{i}$ is the net biomass growth rate of active microbial species $i, i=1, \ldots, N$. $K_{E P S, i}$ denotes the fraction of consumed substrates which is converted in EPS [32]. $k_{d, i}$ is a decay constant and reproduces the natural death of microbial cells [23]. $\mu_{I, i}$ is the specific inactivation rate and models the formation of inactive from active biomass.

The inactive biomass is composed by the cells that experience an adverse habitat. These cells do not have active metabolic function (e.g. uptake of the substrates, production of by-products) and thus do not interact with substrates. The kinetic term modeling the formation of inactive biomass is expressed by

$$
r_{M, i}=\mu_{I, i} f_{i-N}-k_{I 2, i} f_{i}, i=N+1, \ldots, 2 N
$$

where the first term on the right side $\left(\mu_{I, i} f_{i-N}\right)$ denotes the inactivation rate, as in eq. (21), and the second one models the degradation of inactive biomass. The degraded inactive biomass will give rise to the formation of inert material and active dispersed cells. The release of free cells will determine the pore fraction increase. $k_{I 2, i}$ represents the constant decay rate of the inactive microbial components.

Inert biomass results from both the natural decay of the active biomass and the degradation of inactive biomass. According to experimental results, the residual biomass post dispersal shows a slimy and sparse texture and appears to lack viable cells [33] and thus can be considered as inert [1]. Therefore the reaction term is expressed by 


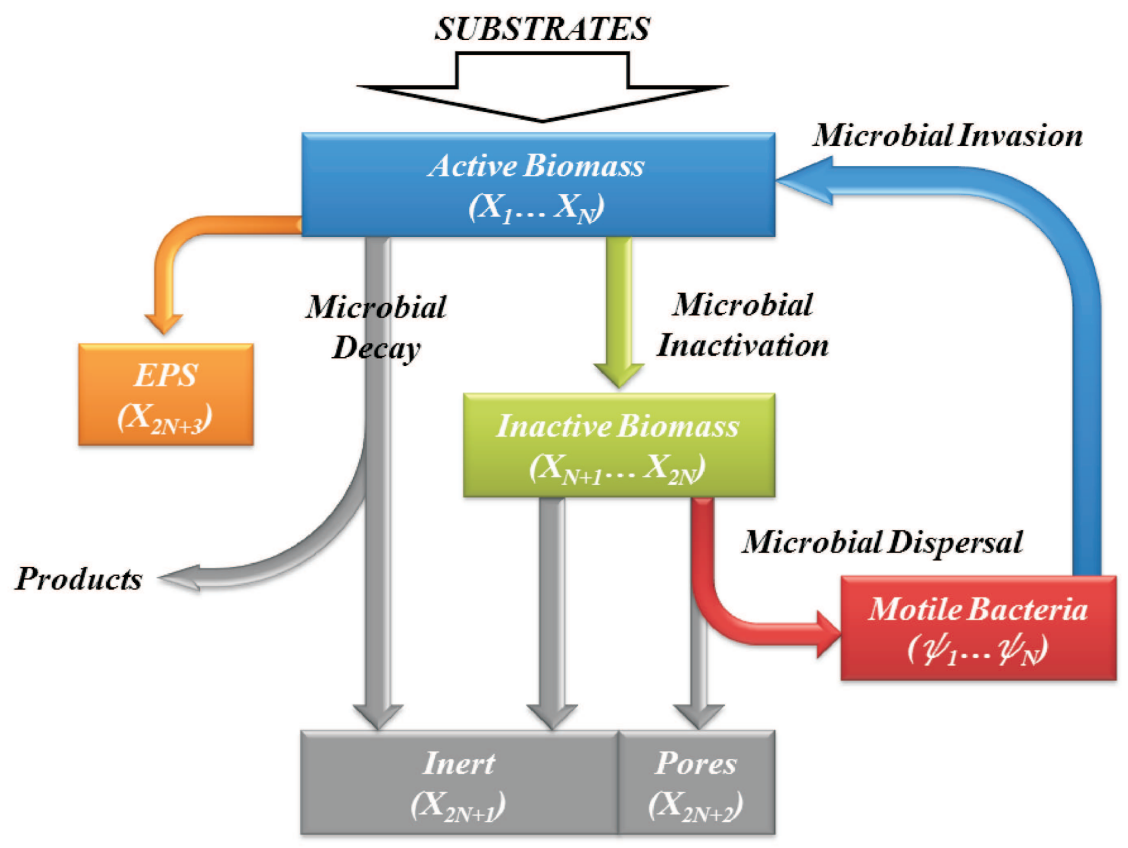

Figure 3. Biofilm pathways. During dispersal, the active biofilm biomass stops its normal metabolic activities and switches to an inactive status. The resulting inactive biomass evolves to inert or to dispersed cells. The formation of voids is modeled by considering a direct dependence of biofilm porosity on the release of free cells. 


$$
r_{M, 2 N+1}=\sum_{i=1}^{N} k_{d, i} f_{i}+\sum_{i=N+1}^{2 N} \alpha_{1, i} k_{I 2, i} f_{i},
$$

where $\sum_{i=1}^{N} k_{d, i} f_{i}$ is the production rate of inert material due to natural decay of active biomasses. The term $\sum_{i=N+1}^{2 N} \alpha_{1, i} k_{I 2, i} f_{i}$ models the formation of inert from inactive biomasses and $\alpha_{1, i}(\leq 1)$ expresses the fraction of inactive biomass which becomes inert.

The formation rate of EPS resulting from the microbial metabolism is

$$
r_{M, 2 N+2}=\sum_{i=1}^{N} K_{E P S, i} \mu_{i} f_{i}
$$

Dispersal influences the biofilm porosity. As mentioned, the residual biomass after dispersal shows a thin texture with higher porosity. The pore volume fraction $f_{2 N+3}$ is assumed to be constituted by two parts having different origins: the first $f_{2 N+3,1}$ represents the innate porosity of the biofilm while the second one $f_{2 N+3,2}$ derives from dispersal. Innate biofilm porosity is modeled by following the approach first introduced by Wanner and Reichert [22] who considered a reaction term for $f_{2 N+3,1}$ such that the production of particulate mass does not alter the innate biofilm porosity. In the present study, the same result is obtained by introducing the following reaction terms for $f_{2 N+3,1}$ and $f_{2 N+3,2}$ in eq. $(16)_{1}$

$$
\begin{gathered}
r_{M, 2 N+3}{ }^{*}=\frac{f_{2 N+3,1}}{1-f_{2 N+3,1}}\left(\sum_{i=1}^{2 N+2} r_{M i}+\sum_{i=N+1}^{2 N} \alpha_{2, i} k_{I 2, i} f_{i}\right), \\
r_{M, 2 N+3}{ }^{* *}=\sum_{i=N+1}^{2 N} \alpha_{2, i} k_{I 2, i} f_{i},
\end{gathered}
$$

where $r_{M, 2 N+3}{ }^{* *}$ represents the production rate for $f_{2 N+3,2}$ due to the dispersal phenomena and $\alpha_{2, i}=1-\alpha_{1, i}$ expresses the fraction of inactive biomass which becomes pores. The overall expression for $f_{2 N+3}$ can be obtained by summing equation (16) $)_{1}$ for $f_{2 N+3,1}$ and $f_{2 N+3,2}$. The derived reaction term takes the form

$$
r_{M, 2 N+3}=\frac{f_{*}}{1-f_{*}}\left(\sum_{i=1}^{2 N+2} r_{M i}+\sum_{i=N+1}^{2 N} \alpha_{2, i} k_{I 2, i} f_{i}\right)+\sum_{i=N+1}^{2 N} \alpha_{2, i} k_{I 2, i} f_{i},
$$

where $f_{*}=f_{2 N+3,1}$, for brevity, represents the innate pore volume fraction which coincides with $f_{2 N+3}(z, 0)$, that is, the porosity of the biofilm before dispersal occurs.

Planktonic species present in the bulk liquid and diffusing into the biofilm may strongly affect biofilm dynamics. Indeed, the free cells might switch their status from planktonic to sessile and vice versa. Planktonic cells can diffuse into the biofilm where they can find a favorable environment for their growth (the invasion phenomenon [24]). This phenomenon actively contributes to biofilm growth through the term $r_{c o l, i}$ in equation $(16)_{1}$, that models the growth of the active biomass $i$ due to the invasion phenomenon, and can assume different forms depending on the problem to be modeled. A specific form will be provided in Section 5.

The two terms $r_{d i s p \psi, i}$ and $r_{c o l \psi, i}$ in equation (19) $)_{1}$ model the production rate of dispersed cells from biofilm during dispersal and the loss of dispersed cells due to invasion respectively. They are expressed by

$$
r_{\text {disp } \psi, i}=\alpha_{2, i} k_{I 2, i} f_{i+N}, r_{c o l \psi, i}=-r_{c o l, i} / Y_{c o l, i}, i=1, \ldots, N,
$$

where $Y_{c o l, i}$ represents the yield of conversion from planktonic to sessile status.

The net conversion rates for substrates in equation $(20)_{1}$ are expressed as $r_{S, j}=\sum_{i=1}^{N} \mu_{i} f_{i} / Y_{i}, j=1, \ldots, m$, with $Y_{i}$ denoting the conversion yield. 


\subsection{Model parameters}

Parameters are listed in Tables $(1,3,5)$. In particular four parameters play a key role. The first one, Inactivation constant $K_{I 1}$, is related to the formation of the inactive biomass when dispersal occurs. According to [1], the phenomenon is quite fast (e.g. a few hours) and for this reason we selected a $K_{I 1}$ value of the same order of magnitude of the maximum growth rate of microbial species. Based on the same reasoning the value of the decay constant for inactive biomass $K_{I 2}$ has been chosen of the same order of magnitude as $K_{I 1}$. The assumption $\alpha_{1, i}+\alpha_{2, i}=1$ follows from a mass balance on inactive biomass and reflects the empirical evidence (see section 1) that during dispersal part of the inactive biomass is released as dispersed cells while the remaining part can be considered to be inert [1]. The loss of mass due to the release of free cells in the surrounding environment determines an increase in biofilm porosity.

\section{Qualitative properties of solutions}

In this section, some properties of the solutions to the free boundary value problem are presented in order to emphasize model consistency and prediction capability.

Proposition 4.1 Let $X_{i}$ be a specific microbial species, whose dynamics are governed by equation (1), rewritten here in terms of $X$ for convenience

$$
\begin{gathered}
\frac{\partial X_{i}}{\partial t}+\frac{\partial}{\partial z}\left(u X_{i}\right)=\rho_{i} R_{M, i}(z, t, \boldsymbol{\psi}, \mathbf{X}, \mathbf{S}), \\
R_{M, i}=r_{M, i}(z, t, \mathbf{f}, \mathbf{S})+r_{c o l, i}(z, t, \psi, \mathbf{S}), 0 \leq z \leq L(t), t>0 .
\end{gathered}
$$

Suppose that

$$
\varphi_{i}(z)=0, r_{M, i}\left|X_{i}=0=0, r_{c o l, i}\right| \psi_{i}=0=0 .
$$

Then equation (29) admits the unique solution $X_{i}=0$.

Proof. The result is obtained from standard arguments.

The hypotheses $(30)_{2,3}$ are usually satisfied for active microbial species (both sessile and planktonic) as shown in equation (21). The expression for $r_{c o l, i}$ will be provided in Section 5 (Equation 43). In addition, note that hypothesis $(30)_{3}$ could be substituted by $r_{c o l, i} \mid \mathbf{S}=0=0$, as the presence of planktonic bacteria alone does not determine the colonization of a new species, indicating that merely the simultaneous presence of substrates and colonizing planktonic species can lead to the growth of sessile bacteria.

Let us focus on the corresponding inactive microbial species denoted by index $i+N$. Note that under the assumption $r_{M, i+N} \mid X_{i}=X_{i+N}=0=0$ and considering $\varphi_{i+N}(z)=0$, the equation for the inactive microbial species admits the unique solution $X_{i+N}=0$. This result also implies absence of dispersal for species $i$, as will be highlighted in the next property.

Proposition 4.2 Let $\psi_{i}$ be the solution of the following initial-boundary value problem

$$
\begin{gathered}
\frac{\partial \psi_{i}}{\partial t}-\frac{\partial}{\partial z}\left(D_{M, i} \frac{\partial \psi_{i}}{\partial z}\right)=r_{\text {disp } \psi, i}(z, t, \mathbf{X}, \mathbf{S})-r_{\text {col } \psi, i}(z, t, \boldsymbol{\psi}, \mathbf{S}), \\
\psi_{i}(z, 0)=0,0 \leq z \leq L(0), \frac{\partial \psi_{i}}{\partial z}(0, t)=0, \psi_{i}(L(t), t)=0,0<z<L(t), t>0,
\end{gathered}
$$

where $i$ is the same index considered in equation (29). Suppose that

$$
\left.r_{\text {disp } \psi, i}\right|_{X_{i}+N=0}=0,\left.\quad r_{\text {col } \psi, i}\right|_{\psi_{i}=0}=0 .
$$

Then the problem (31) admits the unique solution $\psi_{i}=0$.

Proof. The result is obtained from standard arguments.

If a species is not present in the bulk liquid and in the biofilm (either as sessile or planktonic), then it cannot diffuse into the biofilm or further disperse in the bulk liquid. Therefore, in absence of planktonic bacteria, the term $r_{d i s p \psi, i}$ and so the presence of inactive microbial species $X_{i+N}$ is essential for the dispersal and further colonization of species $i$. Note that according to (32) $)_{1}$, the presence of inactive microbial species $X_{i+N}$ does not influence the species 
in planktonic state $\psi_{j}$ with $j \neq i$ as there is a direct correspondence between the active microbial species $X_{i}$ and the related planktonic status $\psi_{i}$.

Proposition 4.3 Consider the initial-value problem (18) and suppose that

$$
\sigma_{a}(t)=0, \quad \sigma_{d}(L(t))=0,\left.\quad \sum_{i=1}^{n} R_{M, i}\right|_{\mathbf{S}=0}=0 .
$$

Then, equation $\left(18_{1}\right)$ admits the unique solution $L=L_{0}$.

Proof. The assumption $(33)_{3}$, with the initial condition $u(0, t)=0$ for equation (3), implies that $u(L(t), t)=0$ and the result is proved.

In absence of mass flux between biofilm and bulk liquid and substrates within the biofilm, the microbial activity stops and the biofilm thickness remains constant. Note that hypothesis $(33)_{1}$ is satisfied for mature biofilms since the attachment biofilm flux has relevance only in the initial growth phase of biofilm. However, $\sigma_{d}(L(t))$ is usually different from zero. In such case, in absence of substrates, biofilm thickness decreases with time.

\section{Applications and numerical solutions}

We apply the model to three illustrative cases. The first one is based on the observations of Shleheck et al. [1] who identified nutrient starvation as the main cause of dispersal in a Pseudomonas Aeruginosa (PA) biofilm. The second shows the dependence of biofilm dispersal on the presence of an inhibiting agent as reported in [9]. The third describes dispersal induced by a self-produced biocide in a $P A$ biofilm. For all model applications, numerical solutions to the free boundary problem stated in Sections 3.2 - 3.5 have been obtained by using the method of characteristics, e.g. $[34,35]$. Accuracy was checked by comparison to the constraint $\sum_{i=1}^{n} f_{i}(z, t)=1$. Simulations were performed using original software developed for this work.

\subsection{Application 1: Pseudomonas aeruginosa dispersal upon starvation}

The first numerical application has been based on the experimental evidence of Schleheck et al. [1]. In their work, the authors performed batch tests on PA biofilms growing on pieces of polyester fleece which were continuously moving in an aerated chemostat inoculated with cultivated PA biomass and fed with a known initial concentration of glucose. They observed that in presence of a significant concentration of glucose, planktonic growth was not detectable within the bulk liquid, while upon glucose limitation (starvation), the PA-attached biofilms dispersed into single cells, resulting in an increase in optical density independent of cellular growth. In addition, it was reported that the biofilm biomass remaining on surface supports exhibited increased amounts of eDNA (the release of genomic DNA, known as extracellular DNA (eDNA) is symptomatic of cell death and lysis phenomena) and dead cells, and appeared to lack viable cells, indicative of dispersal events.

The experiment reported in [1] has been qualitatively reproduced here in silico. A schematic representation of the simulated system is reported in fig (4). The biofilm reactor system used for the experimental tests is characterized by a elevated surface/volume ratio so a $1 D$ biofilm model lends itself well to describe the problem. The model considers the growth and decay of five different components, including PA active biomass $X_{1}=\rho_{1} f_{1}$, inactive biomass resulting from the inactivation of PA biomass $X_{2}=\rho_{2} f_{2}$, inert material $X_{3}=\rho_{3} f_{3}$, EPS $X_{4}=\rho_{4} f_{4}$ and voids $X_{5}=\rho_{5} f_{5}$. Two substrates, glucose $S_{1}$ and oxygen $S_{2}$ are included. As outlined before (Section 3.6), the inactive biomass, the inert material and the EPS are modeled as fictitious microbial species.

Biofilm growth is governed by equations (1), rewritten here for convenience,

$$
\frac{\partial f_{i}}{\partial t}+\frac{\partial}{\partial z}\left(u f_{i}\right)=R_{M, i}(z, t, \psi, \mathbf{X}, \mathbf{S}), 0 \leq z \leq L(t), t>0, i=1,2,3,4,5,
$$

where

$$
R_{M, i}=r_{M, i}(z, t, \boldsymbol{X}, \mathbf{S})+r_{c o l, i}(z, t, \psi, \mathbf{S}) .
$$

The biomass growth rates $r_{M, i}$ are expressed for PA active biomass fraction $f_{1}$ as 


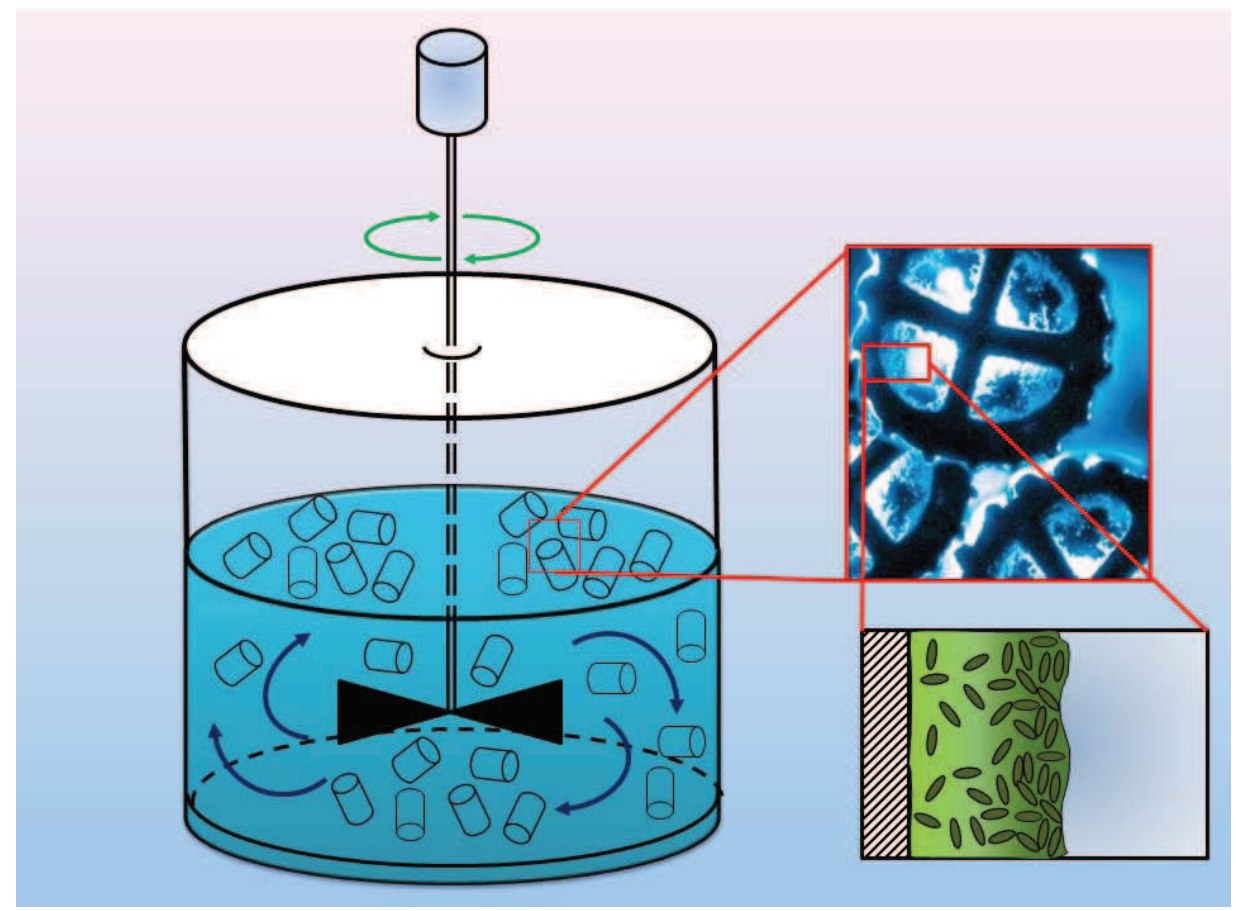

Figure 4. Schematic representation of the simulated system reported in the Application 1. The biofilm grows on the surfaces of suspended carriers, which continuously move thanks to the aeration system. The reactor is operated in batch conditions. The biofilm is modeled as a one-dimensional, continuous, homogeneous system growing in a direction perpendicular to the substratum. 


$$
r_{M, 1}=\left(\left(1-K_{E P S}\right) \mu_{1}(\mathbf{S})-k_{d}-\mu_{I 1}(\mathbf{S})\right) f_{1},
$$

for PA inactive biomass fraction $f_{2}$ as

$$
r_{M, 2}=\mu_{I 1}(\mathbf{S}) f_{1}-\alpha_{1} K_{I 2} f_{2}-\alpha_{2} K_{I 2} f_{2}=\mu_{I 1}(\mathbf{S}) f_{1}-\alpha_{1} K_{I 2} f_{2}-r_{d}
$$

for inert fraction $f_{3}$ as

$$
r_{M, 3}=k_{d} f_{1}+\alpha_{1} K_{I 2} f_{2}
$$

for EPS fraction $f_{4}$ as

$$
r_{M, 4}=K_{E P S} \mu_{1}(\mathbf{S}) f_{1}
$$

and for pore fraction $f_{5}$ as

$$
r_{M, 5}=\frac{f_{*}}{1-f_{*}}\left(\sum_{i=1}^{4} r_{M i}+r_{d}\right)+r_{d} .
$$

The parameters $k_{I 2}, K_{E P S}, \alpha_{1}, \alpha_{2}, f_{*}=f_{5,0}(z)$ have been defined in Section (3.6), while their values assumed for numerical simulations are reported in Tables 1 and 2.

According to [36], double Tessier kinetics have been found to be more appropriate than commonly double Monod kinetics in modelling PA biofilm dynamics. The net biomass growth rates $\mu_{1}$ and the inactivation rate of the active microbial species $\mu_{11}$ are given by

$$
\begin{gathered}
\mu_{1}=\mu_{\max }\left(1-e^{-S_{1} / K s_{1}}\right)\left(1-e^{-S_{2} / K s_{2}}\right), \\
\mu_{I 1}=K_{I 1}\left(\frac{1}{1+S_{1} / I_{1}}\right),
\end{gathered}
$$

following [36], where $\mu_{\max }, K s_{1}, K s_{2}$ are the maximum growth rate, the glucose affinity constant and the oxygen affinity constant for PA biofilm, respectively. $K_{I 1}$ is the inactivation constant and $I_{1}$ is the inhibition constant for the inactivation process (Table 1).

The planktonic species loss rate $r_{c o l, 1}$, due to the invasion phenomena [24] is given by

$$
r_{c o l, 1}=K_{c o l, 1} \frac{\psi_{1}}{k_{\psi, 1}+\psi_{1}}\left(1-e^{-S_{1} / K s_{1}}\right)\left(1-e^{-S_{2} / K s_{2}}\right),
$$

where $K_{c o l, 1}$ is the maximum colonization rate of planktonic species, while $k_{\psi, 1}$ is the kinetic constant for planktonic bacteria (Table 1).

The following initial conditions will be considered for equations (34)

$$
f_{i}(z, 0)=f_{i, 0}(z), 0 \leq z \leq L_{0}, i=1,2,3,4,5 .
$$

The functions $f_{i, 0}(z), i=1, \ldots, 5$, represent the initial volume fractions of biofilm components and their values are reported in Table 2. Diffusion of species in planktonic state $\psi_{1}$ is governed by the equation

$$
\begin{aligned}
\frac{\partial \psi_{1}}{\partial t}-\frac{\partial}{\partial z}\left(D_{M, i} \frac{\partial \psi_{1}}{\partial z}\right) & =r_{d i s p, \psi_{1}}(z, t, \mathbf{X}, \mathbf{S})-r_{c o l, \psi_{1}}(z, t, \boldsymbol{\psi}, \mathbf{S}) . \\
0 & <z<L(t), t>0,
\end{aligned}
$$

The planktonic species loss rate and the planktonic species production rate are

$$
r_{c o l, \psi_{1}}=\frac{r_{c o l, 1}}{Y_{c o l, 1}}=\frac{K_{c o l, 1}}{Y_{c o l, 1}} \frac{\psi_{1}}{k_{\psi, i}+\psi_{1}}\left(1-e^{-S_{1} / K s_{1}}\right)\left(1-e^{-S_{2} / K s_{2}}\right),
$$




$$
r_{\text {disp }, \psi_{1}}=\alpha_{2} k_{I 2} X_{2},
$$

where $Y_{c o l, 1}$ the yield of sessile bacteria on planktonic state (Table 1). The following initial-boundary conditions will be considered for equation (45):

$$
\begin{gathered}
\psi_{1}(z, 0)=0,0 \leq z \leq L_{0}, \\
\frac{\partial \psi_{1}}{\partial z}(0, t)=0, \quad \psi_{1}(L(t), t)=\psi_{1}^{*}(t) \quad 0<t \leq T,
\end{gathered}
$$

where $\psi_{1}^{*}(t)$ satisfies equation (55). The diffusion of substrates is governed by the equations

$$
\frac{\partial S_{j}}{\partial t}-\frac{\partial}{\partial z}\left(D_{S, j} \frac{\partial S_{j}}{\partial z}\right)=r_{S, j}(z, t, \mathbf{X}, \mathbf{S}), j=1,2,
$$

where $D_{S, j}$ denotes the diffusivity coefficient and $r_{S, j}(z, t, \mathbf{X}, \mathbf{S})$ the net conversion rate of substrate $j$. They are expressed by

$$
\begin{aligned}
& r_{S, 1}=-\frac{\mu_{1}(\mathbf{S})}{Y_{1}} X_{1}, \\
& r_{S, 2}=-\frac{\mu_{1}(\mathbf{S})}{Y_{2}} X_{1},
\end{aligned}
$$

for the glucose $S_{1}$ and for the Oxygen $S_{2} . Y_{1}$ and $Y_{2}$ are the yields of PA on glucose and oxygen, respectively. The values assumed for the previous parameters are reported in Tables 1 and 2. The following initial-boundary conditions will be considered for equations (50)

$$
\begin{gathered}
S_{j}(z, 0)=S_{j 0}(z), 0 \leq z \leq L_{0}, j=1,2, \\
\frac{\partial S_{j}}{\partial z}(0, t)=0, \quad S_{1}(L(t), t)=S_{1}^{*}(t), \quad S_{2}(L(t), t)=S_{2 L}, \quad 0<t \leq T,
\end{gathered}
$$

where $S_{1}^{*}(t)$ satisfies equation (56) and $S_{2 L}$ denotes the constant oxygen level within the bulk liquid, whose value is reported in Table 2.

The evolution in time of glucose $S_{1}$ and dispersed cells concentration $\psi_{1}$ in the bulk liquid are set by

$$
\begin{aligned}
& \dot{\psi}_{1}^{*}(t)=-\frac{A}{V} D_{M, 1} \frac{\partial \psi_{1}}{\partial z}(L(t), t), \\
& \dot{S}_{1}^{*}(t)=-\frac{A}{V} D_{S, 1} \frac{\partial S_{1}}{\partial z}(L(t), t),
\end{aligned}
$$

where $V$ and $A$ are the volume of the chemostat and the total biofilm surface area respectively [37]. Equations 55 and 56 are subject to initial conditions

$$
\begin{aligned}
& S_{1}^{*}(0)=\bar{S}_{1}, \\
& \psi_{1}^{*}(0)=\bar{\psi}_{1} .
\end{aligned}
$$

The functions $S_{j 0}(z)$ represent the initial values of substrates within the biofilm. The functions $\psi_{i 0}(z)=0$, represent the initial values of the planktonic species. The functions $\bar{S}_{1}, \bar{\psi}_{1}$ represent the initial concentrations of glucose and planktonic bacteria in the bulk liquid, respectively (Table 2). $L_{0}$ denotes the initial biofilm thickness (Table 2).

The free boundary evolution is governed by equation (15) with initial condition $L(0)=L_{0}$; the velocity $\sigma_{a}(L(t))$ is fixed equal to zero, while $\sigma_{d}(L(t))$ is assumed to be a known function of $L$, in particular 
Table 1. Kinetic parameters used for model simulations of Application 1.

\begin{tabular}{|c|c|c|c|c|}
\hline Parameter & Definition & Unit & Value & References \\
\hline$\mu_{\max }$ & Maximum growth rate of PA01 & $h^{-1}$ & 0.29 & {$[36]$} \\
\hline$K_{E P S}$ & EPS formation by PA01 & $g_{E P S} / g_{\text {biomass }}$ & 0.03 & This study \\
\hline$K s_{2}$ & Oxygen affinity constant for PA01 & $m g l^{-1}$ & 1.18 & {$[36]$} \\
\hline$Y_{1}$ & Yield of PA01 on glucose & $g_{\text {biomass }} / g_{\text {substrate }}$ & 0.628 & {$[36]$} \\
\hline$Y_{2}$ & Yield of PA01 on oxygen & $g_{\text {biomass }} / g_{\text {substrate }}$ & 0.635 & {$[36]$} \\
\hline$K_{d}$ & Microbial decay constant of PA01 & $h^{-1}$ & 0.00021 & This study \\
\hline$K_{I 1}$ & Inactivation constant & $h^{-1}$ & 4.17 & This study \\
\hline$K_{I 2}$ & Decay constant for inactive biomass & $h^{-1}$ & 2.5 & This study \\
\hline$\alpha_{1}$ & Fractionation constant for inactive biomass to inert & - & 0.8 & This study \\
\hline$\alpha_{2}$ & Fractionation constant for inactive biomass to voids & - & 0.2 & This study \\
\hline$I_{1}$ & Inhibition constant for the inactivation process & $m g l^{-1}$ & 0.0005 & This study \\
\hline$K_{c o l, 1}$ & Maximum colonization rate of planktonic species & $h^{-1}$ & $8.3 \cdot 10^{-5}$ & {$[24]$} \\
\hline$k_{\psi, 1}$ & Kinetic constant for planktonic bacteria & $m g l^{-1}$ & $4.1 \cdot 10^{-9}$ & {$[24]$} \\
\hline$Y_{c o l, 1}$ & Yield of planktonic & $g_{\text {planktonic }} / g_{\text {biofilm }}$ & 0.015 & This study \\
\hline$\rho$ & biofilm density & $g m^{-3}$ & 65000 & This study \\
\hline$\lambda$ & Biomass shear constant & $m d^{-1}$ & 150 & This study \\
\hline$D_{S, 1}$ & Diffusion coefficient of Glucose & $m^{2} d^{-1}$ & 0.000073 & This study \\
\hline$D_{S, 2}$ & Diffusion coefficient of Oxygen & $m^{2} d^{-1}$ & 0.000175 & {$[21]$} \\
\hline$D_{M, 1}$ & Diffusion coefficient of planktonic species & $m^{2} d^{-1}$ & 0.000045 & This study \\
\hline$A$ & Reactor surface area & $d m^{2}$ & 54 & This study \\
\hline$V$ & Reactor volume & $d m^{3}$ & 1.5 & This study \\
\hline
\end{tabular}

$$
\sigma_{d}(L(t))=\lambda L^{2}(t)
$$

where $\lambda$ is a shear constant whose value is reported in Table 1 . In the appendix 8 , the model equations in matrix form are shown.

The model outputs are reported in figure 5. Numerical simulations demonstrate model capability of predicting particulate components distribution, substrate and dispersed cells concentration profiles over biofilm depth, biofilm thickness, glucose and planktonic species concentration in the bulk liquid over time. The initial biofilm composition has been defined in Table 2. In particular, the biofilm is set to be initially constituted only by PA active biomass (90\%) and EPS (10\%) with an initial thickness of $0.3 \mathrm{~mm}$; the oxygen concentration in the bulk liquid has been fixed to 7 $\mathrm{mg} / \mathrm{l}$, consistent with continuously aerated systems. The concentrations at $t=0$ of glucose and dispersed cells in bulk liquid are $1.35 \mathrm{mg} / \mathrm{l}$ and 0 , respectively. Note that the biofilm is initially characterized by an innate porosity which is expected to increase over time due to dispersal. We initialize using a 300 um thick biofilm grown with no nutrient limitation and then allow the model to explore how starvation in the interior of the biofilm might influence dispersal.

Figure 5(A1) shows the biofilm distribution and substrate profiles after 6 hours. The biofilm composition is close to the initial one, whereas the glucose concentration in the inner part of the biofilm has already reached zero. Under these conditions, bacteria living in the biofilm experience nutrient starvation, which in turn induces the formation of an inactive biomass, the precursor of dispersal. Even if not visible in the figures (fig. 5(A1)), the overall inactive biomass fraction has already increased from zero to $0.57 \%$ of the total biofilm, mostly located in the inner part of the biofilm where bacterial metabolism is most subject to adverse growing conditions.

After 18 hours, glucose concentration in the biofilm is practically equal to zero everywhere (fig. 5(B2)). The concentration of oxygen remains the same as the value in the bulk liquid throughout the biofilm, confirming the inactivity of the PA biomass. The volume fraction of active biomass is reduced mostly in the inner part, where it is possible to find an increased fraction of inert and inactive biomass (fig. 5(A2)). Dispersal has been initiated by nutrient starvation: it results in the release of dispersed cells, which diffuse through the biofilm and out to the bulk liquid. Indeed, compared to earlier times, the dispersed cells concentration is noticeably different from zero 
Table 2. Initial-boundary conditions for model Application 1.

\begin{tabular}{lclc}
\hline \hline Parameter & Symbol & Unit & Value \\
\hline Initial glucose concentration & $\overline{S_{1}}$ & $\mathrm{mgl}^{-1}$ & 1.35 \\
Oxygen concentration at $L=L(t)$ & $S_{2 L}$ & $\mathrm{mg} l^{-1}$ & 7 \\
Initial planktonic species concentration & $\overline{\psi_{1}}$ & $\mathrm{mg} l^{-1}$ & 0 \\
Time Simulation & $\mathrm{T}$ & $\mathrm{h}$ & 54 \\
Initial Biofilm thickness & $L_{0}$ & $\mathrm{~mm}$ & 0.3 \\
Initial Volume Fraction of PA01 & $f_{1,0}(z)$ & - & 0.85 \\
Initial Volume Fraction of Inactive biomass & $f_{2,0}(z)$ & - & 0.0 \\
Initial Volume Fraction of Inert & $f_{3,0}(z)$ & - & 0.0 \\
Initial Volume Fraction of Pores & $f_{4,0}(z)$ & - & 0.05 \\
Initial Volume Fraction of EPS & $f_{5,0}(z)$ & - & 0.1 \\
Initial Glucose distribution & $S_{1,0}(z)$ & $\mathrm{mg} l^{-1}$ & 0.0 \\
Initial Oxygen distribution & $S_{2,0}(z)$ & $\mathrm{mg} l^{-1}$ & 0.0 \\
Initial $\psi_{1}$ distribution & $\psi_{1,0}(z)$ & $\mathrm{mg} l^{-1}$ & 0.0 \\
\hline \hline
\end{tabular}

throughout the biofilm and higher in the deepest layers of the microbial mass where dispersal started (fig. 5(A2)).

Inert material originates from both the natural decay of active biomass and the disintegration of inactive material: its concentration remains higher in the inner part of the biofilm, where the dispersal phenomenon was initiated and continuously affects biofilm dynamics. Inactive biomass derives from the inactivation of PA biomass with a rate that is a function of the glucose concentration within the biofilm. As expected, its concentration is higher in the innermost layers of the biofilm but remains lower than the inert concentration, to which it converts at a constant rate. The rest of the inactive biomass disperses leading to the formation of voids which contributes to increased biofilm porosity.

In Figure 5(A3) it can be seen that dispersal, which was initiated in the inner part of the biofilm, has now affected the whole microbial mass. The concentration of active microbial species has further decreased in the outmost layers while inert and inactive biomass further increase in the inner part. Biofilm porosity is found to increase throughout the biofilm, confirming the loss of biomass due to the release of dispersed cells (fig. 5(A3)). The concentration of the latter is higher than in earlier simulation times. No differences in glucose and oxygen profiles can be seen (fig. 5(B3)).

Computations consist of 54h of simulation time. Convergence to a steady state configuration occurs after approximately 42 hours, at which time the biofilm is constituted of only inert material, EPS and voids. This is due to the fact that all the active biomass has converted in inactive due to nutrient starvation; inactive biomass in turn has been dispersed which has led to loss of free dispersed cells from the microbial mass with accompanying formation of inert material and increase in biofilm porosity (fig. 5(A4)). According to mass balance, biofilm porosity reaches a constant value in depth depending on initial constant biomass fractions. The concentration of oxygen remains at $7 \mathrm{mg} / \mathrm{l}$ throughout the biofilm while glucose has been totally consumed by the system (figs 5(B4)). Time course data from 42 to 54 hours are not shown.

In Figure 6, we show cells and glucose concentrations in the bulk liquid over time. Glucose concentration decreases with time as it is continuously consumed by the active biomass growing in the batch system, approaching zero at 15 hours. As soon as the glucose is depleted both in the biofilm and in the bulk liquid, dispersal starts and cells are released into the bulk liquid, with concentration approaching stationary value of approximately $0.5 \mathrm{mg} / \mathrm{l}$. Profiles obtained by numerical simulations show the same trends of the experimental results reported in [1] (see figure $5 \mathrm{~A}$ in [1]).

Note in addition, also as reported in [1], by analyzing the biofilm structure after dispersal, the remaining biomass exhibited increased eDNA and dead cells, and appeared to lack viable cells, all indicative of dispersal events This implies that Surface-attached biofilm growth predominates with favorable environmental conditions (presence of glucose), while the biofilms disperses into the planktonic phase when not favourable environmental condition occurs (glucose starvation). The biofilm configuration after dispersal is comparable with simulation results. Indeed, as reported in Figure 5A4, the biofilm is constituted by inert material and EPS and without the presence of active biomass. 
(A1)

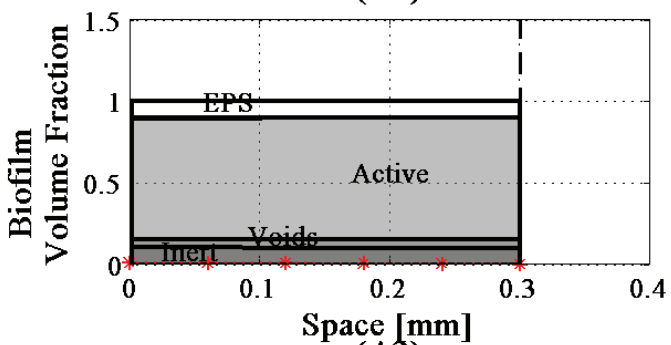

(A2)

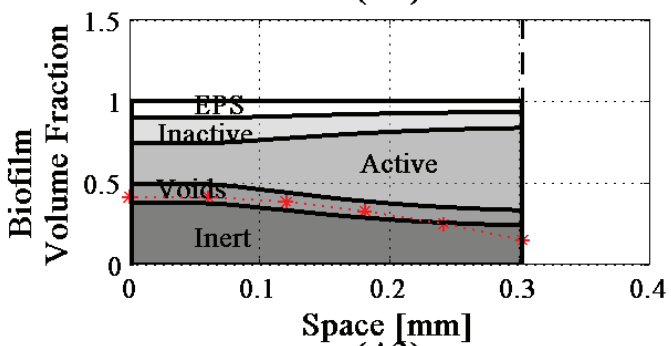

(A3)

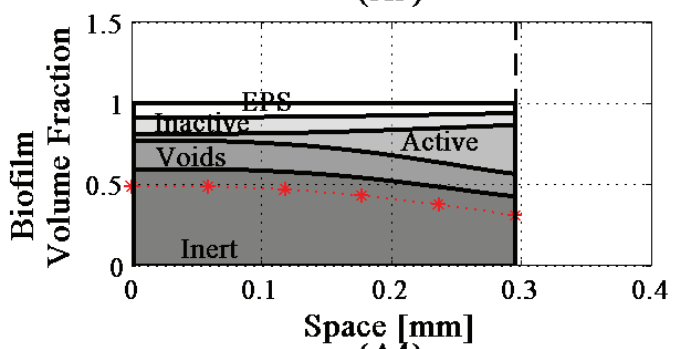

(A4)

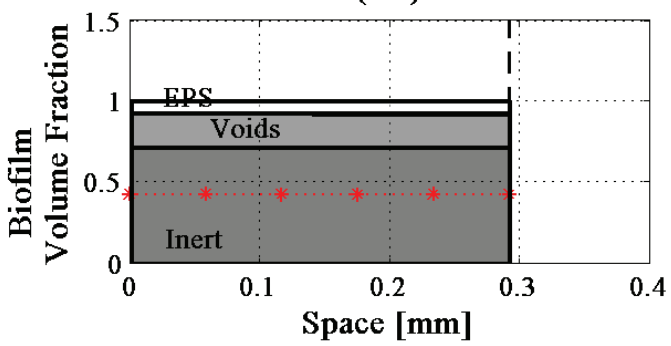

(B1)

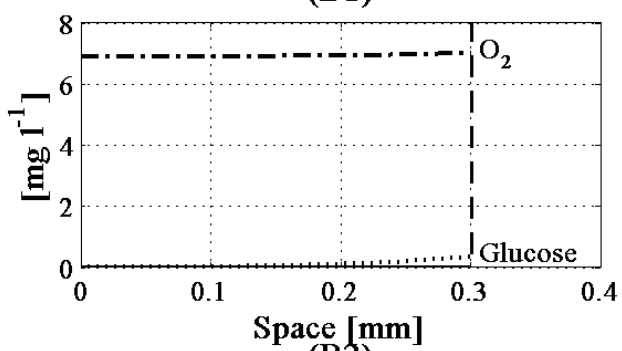

(B2)

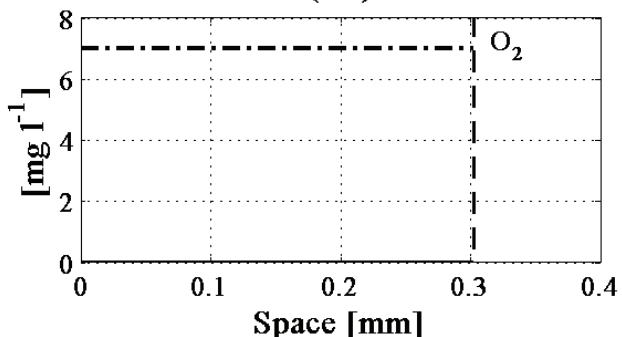

(B3)

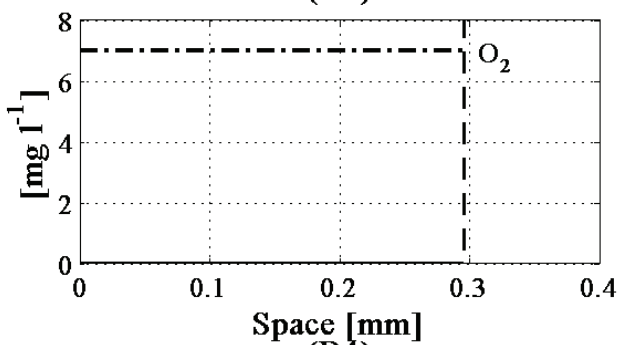

(B4)

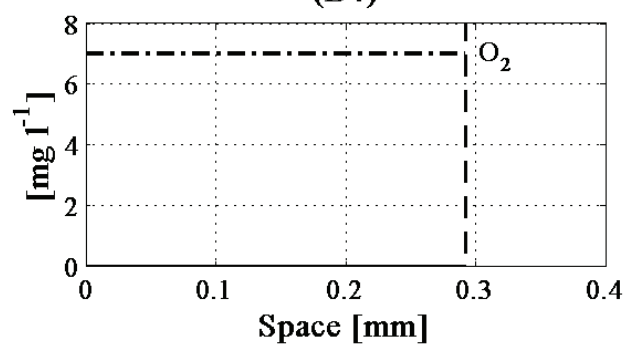

Figure 5. Effect of Starvation-induced dispersal of a PA biofilm on bacterial volume fractions, $\psi_{1}$ profile (dotted-starred red line) and substrate concentration profiles in a batch system after 6 (A1,B1), 18 (A2,B2), 24 (A3,B3), 42 (A4,B4) hours. Simulation results refer to Application 1. Note that glucose concentration reaches too low values to be visible over the biofilm after 6 hours of simulation time. 


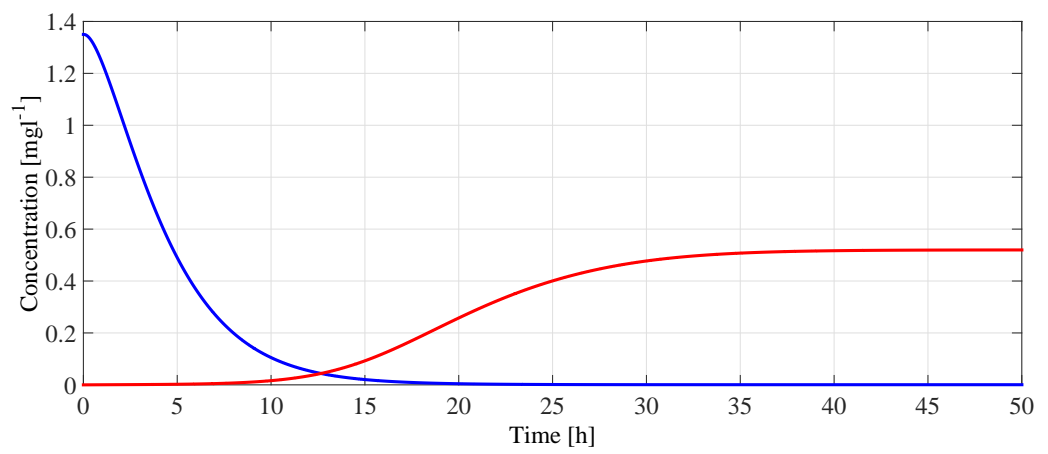

Figure 6. Dispersed cells and glucose profiles in the bulk liquid over 50 hours time simulation. (red line) $\psi_{1} *$, dispersed cells profile. (blue line) Glucose profile. Simulation results refer to Application 1.
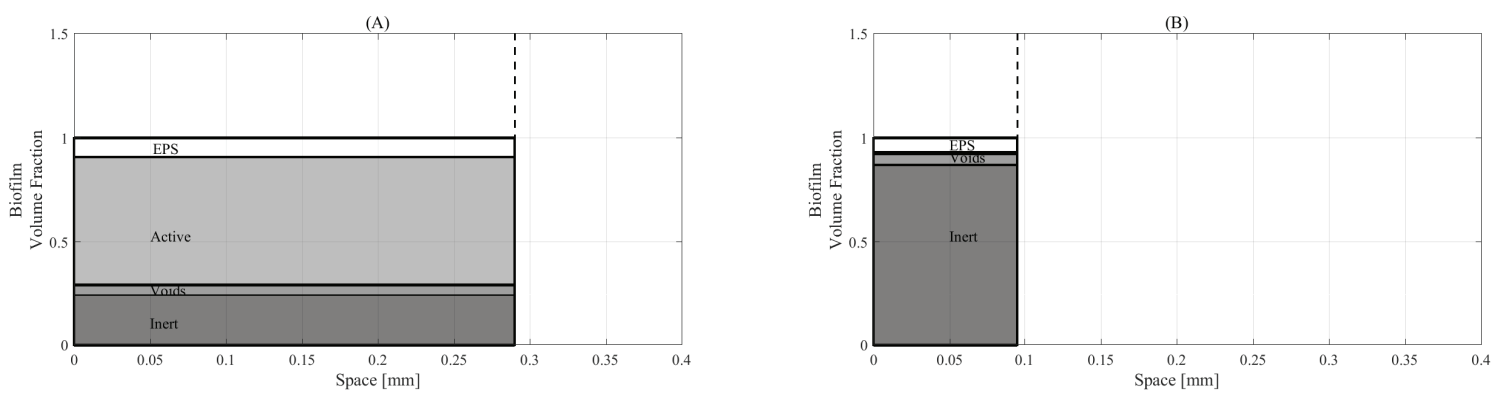

Figure 7. Biofilm volume fractions after 54 hours (A) and 600 hours (B) in a multispecies biofilm growing in a batch system and experiencing nutrient starvation. Simulation results are obtained by using the Wanner-Gujer biofilm model and assuming the same initial and boundary conditions of Application 1

If we consider a lower initial biofilm thickness $50 \mu \mathrm{m}$ (results not shown) the glucose goes to zero in more time respect to the case of initial biofilm of $300 \mu \mathrm{m}$ (Figure 5). A substantial biofilm growth (from 50 to $76 \mathrm{~m}$ ) occurs in the first days of the simulation until the complete depletion of glucose.

To highlight model capability of reproducing dispersal phenomenon we reproduce the same biological case presented in sec.(5.1) with a model that does not account for biofilm dispersal. To this aim, we consider the Wanner based model presented in this study with $r_{\text {disp }}=\mu_{I}=0$. After approximately 54 hours, the active biomass stops growing and slowly converts to inert material. The simulations shows that though glucose substrate is completed depleted, nevertheless biofilm structure is characterized by significant presence of active biomass (fig.7,(A)). This is because the decay rate is low and not suitable to model a phenomenon that, as reported in experimental evidence [1], takes place in few hours. We then run the simulation for more time and as expected the active biomass volume fraction decreases and becomes zero according to the reaction kinetics. However, the biofilm assumes a different steady-state configuration compared to the dispersal model (fig.5,(A4)). Indeed the pore volume fraction is smaller in comparison to the proposed model and as expected the biofilm is mainly constituted by inert material (fig.7,(B)). Due to the long time needed to reach a biofilm constituted only by inert material, erosion results in a significant reduction in biofilm thickness.

\subsection{Application 2: Modeling dispersal induced by a non-lethal antibiofilm agent}

In this example, the model is applied to the case of PA biofilm dispersal triggered by the presence of an antibiofilm agent that, as a biocide-free strategy, does not exert lethal effects on active microbial cells but rather it interferes with 
their propensity to follow a sessile lifestyle, inducing in most of the cases the release of planktonic cells from the microbial mass, i.e., dispersal [9]. Thus, in this example, the PA biofilm is assumed to grow in a system constantly fed with glucose and continuously aerated, and, when the biofilm reaches a stationary state at $t=t_{1}$, an antibiofilm agent is supplied to the system. Reaction of antibiofilm agent with active biomass results in formation of the inactive biomass, which precedes the dispersal phenomenon. The same biofilm components, $f_{i}, i=1, \ldots, 5$, as in the previous application case are considered (Table 2). However glucose $S_{1}$ and oxygen $S_{2}$ are now provided continuously to the system. An antibiofilm agent $\theta$ is supposed to reach the surface of the biofilm at $t=t_{1}$ and then begins to diffuse into the biofilm. Biofilm dynamics are governed by equations (34)-(44). Equation (42) for the inactivation rate is replaced by:

$$
\mu_{I 1}=K_{I 1}\left(\frac{\theta}{K_{\theta}+\theta}\right),
$$

where $K_{I 1}$ still represents the inactivation constant for active microbial species $f_{1}$ and $K_{\theta}$ denotes the half saturation constant of species $f_{1}$ on $\theta$. The fate of dispersed cells within the biofilm and in the bulk liquid is modeled by equations (45)-(49), (55), (58). Glucose and oxygen dynamics are governed by equations (50)-(54)1,3. Condition $(54)_{2}$ is replaced by $S_{1}(L(t), t)=S_{1 L}$, where $S_{1 L}$ is the time-independent glucose concentration in the bulk liquid. The values assumed for $S_{1 L}$ and $S_{2 L}$ in numerical simulations are provided in Table 3. Diffusion and consumption of $\theta$ are modeled as

$$
\frac{\partial \theta}{\partial t}-\frac{\partial}{\partial z}\left(D_{\theta} \frac{\partial \theta}{\partial z}\right)=r_{\theta}(z, t, \mathbf{X}, \theta)
$$

where $D_{\theta}$ and $r_{\theta}$ are the diffusion coefficient (Table 3) and the conversion rate of $\theta$. The latter is expressed by

$$
r_{\theta}=-\frac{\mu_{I 1}}{Y_{\theta}} X_{1},
$$

where $Y_{\theta}$ denotes the conversion yield (Table 3). We set initial-boundary conditions for equation (61) to be

$$
\begin{gathered}
\theta(z, 0)=\theta_{0}(z), 0 \leq z \leq L_{0}, \\
\frac{\partial \theta}{\partial z}(0, t)=0, \theta(L(t), t)=\theta_{L}(t), 0<t \leq T, \theta_{L}(t)=\left\{\begin{array}{l}
0,0<t \leq t_{1}, \\
>0, t_{1}<t \leq T,
\end{array}\right.
\end{gathered}
$$

where the function $\theta_{0}(z)$ is the initial distribution of $\theta$ within the biofilm at $t=t_{0}$ and $\theta_{L}(t)$ is the antibiofilm agent concentration in the bulk liquid. Note in equation (64) that the concentration of $\theta$ on the free boundary is a step function defined by $\theta_{L}=0$ for for $t \leq t_{1}$, and for $t \geq t_{1}, \theta_{L}$ is equal to the constant value reported in table 4 . The free boundary evolution is governed by equations (15),(59) with initial condition $L(0)=L_{0}$. The values of $L_{0}, t_{1}$ and $\lambda$ used in numerical simulations are reported in Tables 3 and 4 , for all the other parameters the values are reported in Tables 1 and 2.

The diffusion-reaction of planktonic state $\psi_{1}$ is governed by the equation

$$
\frac{\partial \psi_{1}}{\partial t}-\frac{\partial}{\partial z}\left(D_{M, 1} \frac{\partial \psi_{1}}{\partial z}\right)=r_{d i s p, \psi_{1}}(z, t, \mathbf{X}, \mathbf{S}) .0<z<L(t), t>0,
$$

with species production rate

$$
r_{d i s p, \psi_{1}}=\alpha_{2} k_{I 2} X_{2}
$$

The following initial-boundary conditions will be considered for equation (65):

$$
\begin{gathered}
\psi_{1}(z, 0)=0,0 \leq z \leq L_{0}, \\
\frac{\partial \psi_{1}}{\partial z}(0, t)=0, \quad \psi_{1}(L(t), t)=\psi_{1}^{*} t \quad 0<t \leq T,
\end{gathered}
$$


Table 3. Kinetic parameters used for model simulations of Application 2.

\begin{tabular}{llccc}
\hline \hline Parameter & Definition & Unit & Value & References \\
\hline$Y_{\theta}$ & like-yield constant of PA on $\theta$ & $g_{\text {biomass }} / g_{\text {substrate }}$ & 10 & This study \\
$K_{\theta}$ & like half saturation constant of PA on $\theta$ & $m g l^{-1}$ & 4.17 & This study \\
$D_{\theta}$ & Diffusion coefficient of $\theta$ & $m^{2} d^{-1}$ & 0.00015 & This study \\
$\lambda$ & Biomass shear constant & $m m h^{-1}$ & 50 & This study \\
\hline \hline
\end{tabular}

Table 4. Initial-boundary conditions for model Application 2.

\begin{tabular}{lclc}
\hline \hline Parameter & Symbol & Unit & Value \\
\hline Glucose concentration at $L=L(t)$ & $S_{1 L}$ & $\mathrm{mg} l^{-1}$ & 5 \\
Oxygen concentration at $L=L(t)$ & $S_{2 L}$ & $\mathrm{mg} l^{-1}$ & 7 \\
$\theta$ concentration at $L=L(t)$ & $\theta_{L}$ & $\mathrm{mg} l^{-1}$ & 5 \\
Time Simulation & $\mathrm{T}$ & $\mathrm{h}$ & 336 \\
Initial Biofilm thickness & $L_{0}$ & $\mathrm{~mm}$ & 0.3 \\
Time of inhibitor injection & $t_{1}$ & $\mathrm{~h}$ & 24 \\
Initial $\theta$ distribution & $\theta_{0}(z)$ & $\mathrm{mg} l^{-1}$ & 0.0 \\
\hline \hline
\end{tabular}

where $\psi_{1}^{*}(t)$ is the constant concentration in the bulk liquid.

In the appendix 9, the model equations in matrix form are shown. The results of numerical simulations for Application 2 are reported in Figures 8. In Figure 8(A1) the spatial distribution of particulate components and the substrate profiles within biofilm after $24 \mathrm{~h}$ of simulation time are shown. The biofilm has reached a steady-state composition: the active biomass represents the most abundant component due to the high availability of nutrients; the remaining part of the biofilm is constituted by EPS (second highest volume fraction), inert material, which forms from the natural decay of active bacteria, and voids filled with liquid (Fig. 8(A1)). Glucose and oxygen are characterized by stationary and fully penetrated profiles. At this point, an antibiofilm agent is supplied to the system, where it diffuses into and reacts with the active biomass.

Figures 8(A2)-(B2) show biofilm composition and substrate profiles after 42 hours of simulation time. The active biomass volume fraction is reduced mostly in the outer biofilm where inert and inactive biomass are increased (fig. 8(A2)). This is mostly due to the fact that dispersal is initiated in the outer areas where the concentration of $\theta$ is higher. The direct dependence of the inactivation rate on the concentration of the antibiofilm agent is consistent with this distribution of active and inactive biomass. Similarly, inert material shows a higher concentration near the surface of the biofilm and represents, after the active biomass, the most abundant component as it is both produced through natural decay and dispersal. The presence of dispersed cells demonstrates that dispersal has already occurred and part of the inactive biomass has been loss in planktonic form. The loss of biomass contributes to increase biofilm porosity (fig. 8(A2)). Both oxygen and glucose present a fully penetrated profile (fig. 8(B2)). The antibiofilm agent $\theta$ is characterized, in contrast, by a penetration limited profile due to its reaction with the active biomass.

In figure $8(\mathrm{~A} 3)$ it is possible to observe a significant reduction in active biomass in the external part of the biofilm where its concentration approaches zero. Inert material is present throughout the biofilm and predominates near the surface. Inactive biomass is significantly reduced everywhere due to its continuous release in the form of planktonic cells and to its transformation into inert material. The increase in pore volume fraction throughout the biofilm confirms the loss of biomass due to the release of dispersed cells (fig. 8(A3)). Due to inactivation induced by the antibiofilm agent, glucose concentration increases as it is only consumed in the inner layers where the biomass is still active and has not reacted with the antibiofilm agent. Note that antibiofilm agent is still transport limited. Oxygen concentration presents the same trend as the glucose concentration and is characterized by a fully-penetrated profile (fig. 8(B3)).

Glucose and oxygen concentrations are increased throughout the biofilm when compared to previous simulation times. This is due to inactivation of cell metabolisms as a consequence of the presence of sub-critical antibiofilm agent. However, the oxygen and glucose concentrations within the biofilm are still lower than the value assumed in 
(A1)

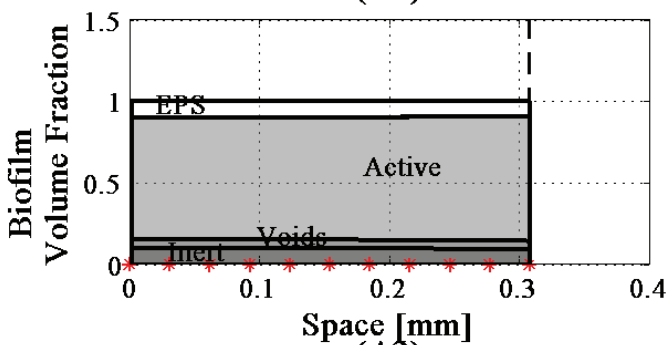

(A2)

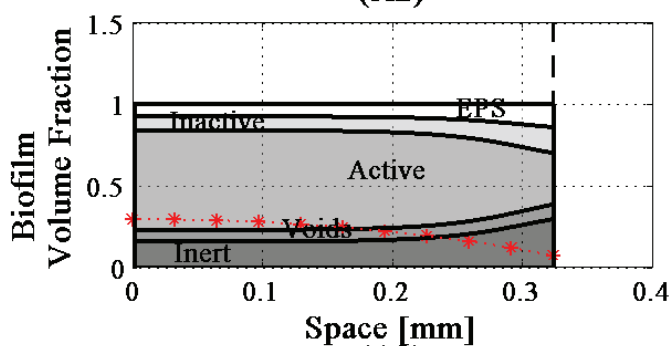

(A3)

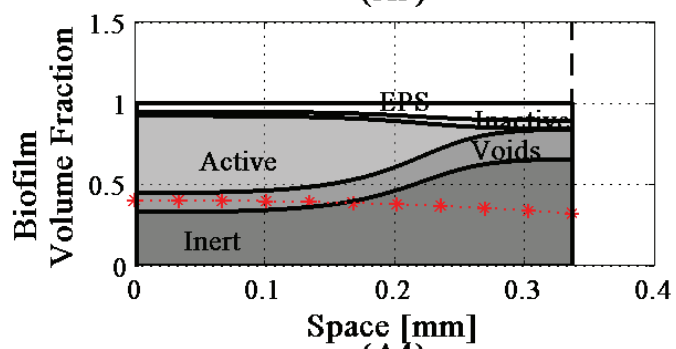

(A4)

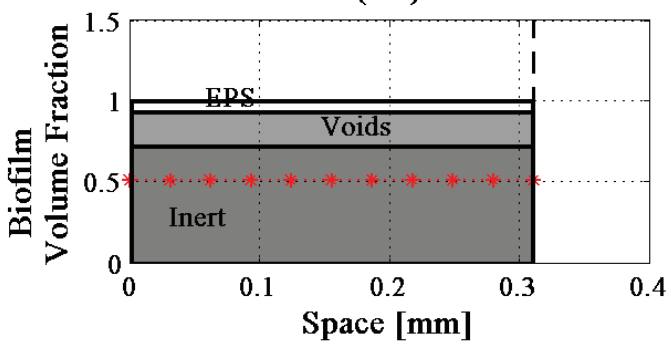

(B1)

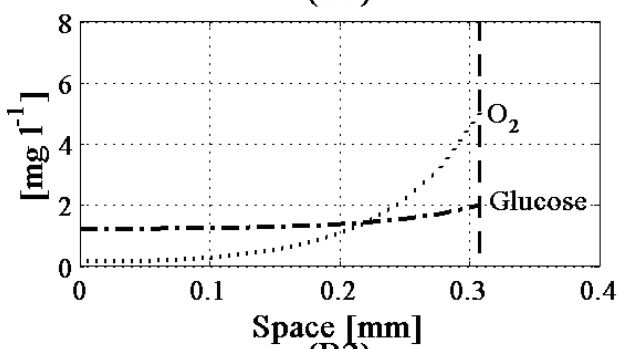

(B2)

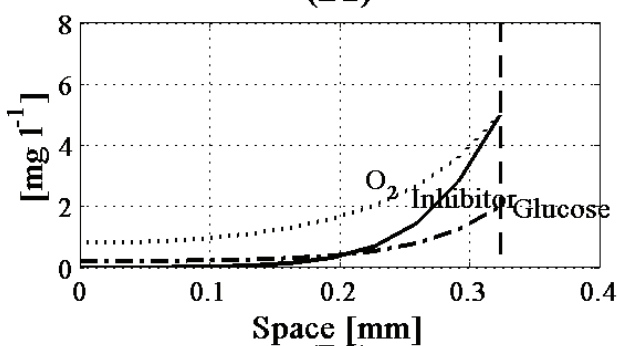

(B3)

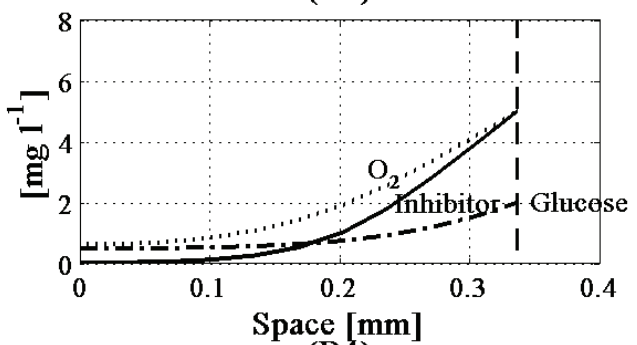

(B4)

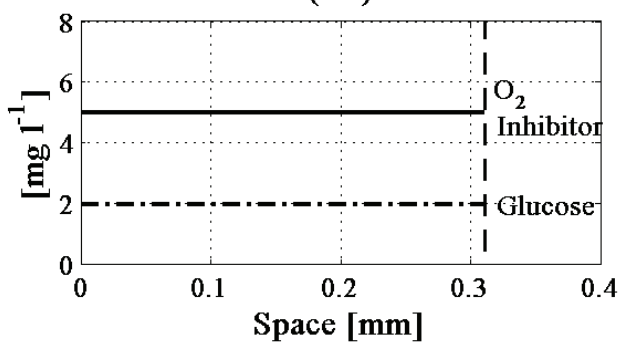

Figure 8. Dispersal phenomenon induced by the diffusion of an antibiofilm agent in non-lethal concentration: bacterial volume fractions, $\psi_{1}$ profile (dotted-starred red line) and substrate concentration profiles after 24 (A1,B1), 42 (A2,B2), 96 (A3,B3), 336 (A4,B4) hours. Simulation results refer to Application 2. 
the bulk liquid as they are consumed by the remaining fraction of active biomass at the bottom of the biofilm.

After 336 hours of simulation time, the biofilm reaches a steady configuration constituted only by inert material, EPS and voids. This particulate distribution resembles the stationary configuration obtained in the previous simulation set, demonstrating that both nutrient starvation and the presence of a non-lethal antibiofilm agent result in the temporary "death" of the biofilm and its dispersal through the release of planktonic cells. As seen in figure 8(A4), all the active biomass has been converted into inert material after transiting the intermediate inactive state, which in turn is subject to dispersal. This leads to the loss of free planktonic cells from the microbial mass with consequent increase in biofilm porosity (fig. 8(A4)). For all the dissolved components, the concentration in each point of the biofilm is equal to the value assumed in the bulk liquid. These constant profiles confirms the absence of microbial metabolism within the biofilm (fig. 8(B4)). As expected, inert material represents the most abundant component throughout the biofilm.

\subsection{Application 3: Modeling dispersal induced by a self-produced biocide agent}

In this example, the model is applied to PA biofilm dispersal triggered by the formation of a self-produced biocide agent. The biofilm is constituted by PA bacteria which oxidize organic matter under aerobic regimes. In the inner part of the biofilm, bacterial respiration can induce the formation of an anoxic zone. Under anoxic conditions PA bacteria can switch their metabolism and, in presence of nitrate $\left(\mathrm{NO}_{3}^{-}\right)$, can perform denitrification [29, 30]. During denitrification, nitrate is reduced to nitrite $\left(\mathrm{NO}_{2}^{-}\right)$first, and then nitric oxide $(\mathrm{NO})$. $N O$ is ultimately reduced to nitrogen gas $N_{2}$ (via $N_{2} O$ ). Experimental results $[29,30]$ have shown that temporary accumulation of NO can occur in the transition zone between the aerobic and anoxic regions and induce dispersal. Here we consider a biofilm of depth $60 \mu \mathrm{m}$ fed with a constant concentration of $1000 \mathrm{mg} / \mathrm{L}$ of organic matter (glucose, expressed as COD equivalent in the simulation), $7 \mathrm{mg} / \mathrm{L}$ oxygen and $25 \mathrm{mg} / \mathrm{L}$ nitrate. $\mathrm{NO}_{3}^{-}$diffuses into the biofilm and in the inner part (anoxic zone) is reduced to $\mathrm{NO}_{2}^{-}$and further to $\mathrm{NO}$. The accumulation of $\mathrm{NO}$ induces inactivation leading subsequently to dispersal. Biofilm components considered in this application $\left(f_{i}, i=1, \ldots, 4\right)$ are: PA active biomass $X_{1}=\rho_{1} f_{1}$, inactive biomass resulting from the inactivation of PA biomass $X_{2}=\rho_{2} f_{2}$, inert material $X_{3}=\rho_{3} f_{3}$, and voids $X_{4}=\rho_{4} f_{4}$. Five substrates, glucose $S_{1}$, oxygen $S_{2}$, nitrate $S_{3}$, nitrite $S_{4}$ and nitric oxide $S_{5}$, are tracked.

Biofilm growth is governed by equations (34)-(35), where the biomass growth rates $r_{M, i}$ are expressed as

$$
\begin{gathered}
r_{M, 1}=\left(\mu_{1}(\mathbf{S})-k_{d}-\mu_{I 1}(\mathbf{S})\right) f_{1}, \\
r_{M, 2}=\mu_{I 1}(\mathbf{S}) f_{1}-\alpha_{1} K_{I 2} f_{2}-\alpha_{2} K_{I 2} f_{2}=\mu_{I 1}(\mathbf{S}) f_{1}-\alpha_{1} K_{I 2} f_{2}-r_{d} \\
r_{M, 3}=k_{d} f_{1}+\alpha_{1} K_{I 2} f_{2}, \\
r_{M, 4}=\frac{f_{*}}{1-f_{*}}\left(\sum_{i=1}^{4} r_{M i}+r_{d}\right)+r_{d},
\end{gathered}
$$

for PA active biomass $f_{1}$, PA inactive biomass $f_{2}$, inert $f_{3}$ and pores fraction $f_{5}$, respectively.

The parameters $k_{I 2}, \alpha_{1}, \alpha_{2}, f_{*}=f_{5,0}(z)$ have been defined in Section (3.6), while their values assumed for numerical simulations are reported in Tables 1 and 2 .

The net biomass growth rate $\mu_{1}$ and the inactivation rate $\mu_{I 1}$ of the active microbial species are given by

$$
\mu_{1}=\mu_{1,1}+\mu_{1,2}+\mu_{1,3}+\mu_{1,4}
$$

with:

$$
\begin{gathered}
\mu_{1,1}=\mu_{\max , 1} \frac{S_{1}}{K_{S_{1}}+S_{1}} \frac{S_{2}}{K_{S_{2}}+S_{2}} \\
\mu_{1,2}=\beta_{1} \mu_{\max , 1} \frac{S_{1}}{K_{S_{1}}+S_{1}} \frac{S_{3}}{K_{S_{3}}+S_{3}} \frac{K_{\text {in }}}{K_{\text {in }}+S_{2}},
\end{gathered}
$$




$$
\begin{gathered}
\mu_{1,3}=\beta_{2} \mu_{\max , 1} \frac{S_{1}}{K_{S_{1}}+S_{1}} \frac{S_{4}}{K_{S_{4}}+S_{4}} \frac{K_{\text {in }}}{K_{\text {in }}+S_{2}}, \\
\mu_{1,4}=\beta_{3} \mu_{\max , 1} \frac{S_{1}}{K_{S_{1}}+S_{1}} \frac{S_{5}}{K_{S_{5}}+S_{5}} \frac{K_{\text {in }}}{K_{\text {in }}+S_{2}}, \\
\mu_{I 1}=K_{I 1} \frac{S_{5}^{n}}{K_{N O}^{n}+S_{5}^{n}},
\end{gathered}
$$

where $\mu_{\max , i}, K_{S, j}$, are the maximum growth rates, and the substrate affinity constants for PA biofilm, respectively [38]. $K_{\text {in }}$ represents the oxygen inhibition constant while $K_{I 1}$ is the inactivation constant and $K_{N O}$ denotes the half saturation constant of species $f_{1}$ on $N O$. The values assumed for numerical simulations are reported in Tables3.

The diffusion-reaction of planktonic state $\psi_{1}$ is governed by the equation

$$
\frac{\partial \psi_{1}}{\partial t}-\frac{\partial}{\partial z}\left(D_{M, 1} \frac{\partial \psi_{1}}{\partial z}\right)=r_{d i s p, \psi_{1}}(z, t, \mathbf{X}, \mathbf{S}) .0<z<L(t), t>0,
$$

with species production rate

$$
r_{d i s p, \psi_{1}}=\alpha_{2} k_{I 2} X_{2},
$$

The following initial-boundary conditions will be considered for equation (79):

$$
\begin{gathered}
\psi_{1}(z, 0)=0,0 \leq z \leq L_{0}, \\
\frac{\partial \psi_{1}}{\partial z}(0, t)=0, \quad \psi_{1}(L(t), t)=\psi_{L} \quad 0<t \leq T,
\end{gathered}
$$

where $\psi_{L}$ is the constant concentration in the bulk liquid (Table 6).

Glucose, oxygen, nitrate and nitrite dynamics are governed by equations (50) where the net conversion rates $r_{S, j}(z, t, \mathbf{X}, \mathbf{S})$ for substrates $j=1, . ., 5$ are

$$
\begin{gathered}
r_{S 1}=-\frac{\mu_{1}(\mathbf{S})}{Y_{1}} X_{1}, \\
r_{S 2}=\left(1-\frac{1}{Y_{1}}\right) \mu_{1,1}(\mathbf{S}) X_{1}, \\
r_{S 3}=\left(1-\frac{1}{Y_{1}}\right) \frac{1}{1.14} \mu_{1,2}(\mathbf{S}) X_{1}, \\
r_{S 4}=-\left(1-\frac{1}{Y_{1}}\right) \frac{1}{1.14} \mu_{1,2}(\mathbf{S}) X_{1}+\left(1-\frac{1}{Y_{1}}\right) \frac{1}{0.58} \mu_{1,3}(\mathbf{S}) X_{1}, \\
r_{S 5}=-\left(1-\frac{1}{Y_{1}}\right) \frac{1}{0.58} \mu_{1,3}(\mathbf{S}) X_{1}+\left(1-\frac{1}{Y_{1}}\right) \frac{1}{1.14} \mu_{1,4}(\mathbf{S}) X_{1},
\end{gathered}
$$

The following initial-boundary conditions will be considered for equations (50):

$$
\begin{gathered}
S_{j}(z, 0)=S_{j 0}(z), 0 \leq z \leq L_{0}, j=1, \ldots, 5 . \\
\frac{\partial S_{j}}{\partial z}(0, t)=0, \quad S_{j}(L(t), t)=S_{j L}, \quad j=1, \ldots, 5, \quad 0<t \leq T,
\end{gathered}
$$

where $S_{j L}$ denotes the substrate levels within the bulk liquid, whose value is reported in Table 6 . The free boundary evolution is governed by equations (15),(59) with initial condition $L(0)=L_{0}$. The values of $L_{0}$ and $\lambda$ used in numerical simulations are reported in Tables 5 and 6 , for all the other parameters the values are reported in Tables 1 and 2. 
Table 5. Kinetic parameters used for model simulations of Application 3.

\begin{tabular}{|c|c|c|c|c|}
\hline Parameter & Definition & Unit & Value & References \\
\hline$\mu_{\max , 1}$ & Maximum growth rate of PA01 & $h^{-1}$ & 0.29 & [36] \\
\hline$K s_{1}$ & COD affinity constant for PA01 & $m g l^{-1}$ & 26.9 & [36] \\
\hline$K s_{2}$ & Oxygen affinity constant for PA01 & $m g l^{-1}$ & 1.18 & [36] \\
\hline$K s_{3}$ & Nitrate affinity constant for PA01 & $m g l^{-1}$ & 1.18 & This study \\
\hline$K s_{4}$ & Nitrite affinity constant for PA01 & $m g l^{-1}$ & 1.18 & This study \\
\hline$K s_{5}$ & NO affinity constant for PA01 & $m g l^{-1}$ & 1.18 & This study \\
\hline$Y_{1}$ & yield of PA & $g_{\text {biomass }} / g_{\text {substrate }}$ & 10 & This study \\
\hline$K_{N O}$ & like half saturation constant of PA on $\mathrm{NO}$ & $m g N O / L$ & 0.95 & This study \\
\hline$\beta_{1}$ & Reduction factor for denitrification $\mathrm{NO}_{3}-\mathrm{NO}_{2}$ & - & 0.5 & This study \\
\hline$\beta_{1}$ & Reduction factor for denitrification $\mathrm{NO}_{2}-\mathrm{NO}$ & - & 0.7 & This study \\
\hline$\beta_{1}$ & Reduction factor for denitrification $\mathrm{NO}-\mathrm{N}$ & - & 0.9 & This study \\
\hline$D_{1}$ & Diffusion coefficient of $C O D$ & $m^{2} d^{-1}$ & 0.000253 & This study \\
\hline$D_{2}$ & Diffusion coefficient of Glucose & $m^{2} d^{-1}$ & 0.000375 & This study \\
\hline$D_{3}$ & Diffusion coefficient of Nitrate & $m^{2} d^{-1}$ & 0.000375 & This study \\
\hline$D_{4}$ & Diffusion coefficient of Nitrite & $m^{2} d^{-1}$ & 0.000375 & This study \\
\hline$D_{5}$ & Diffusion coefficient of NO & $m^{2} d^{-1}$ & 0.000675 & This study \\
\hline$K_{\text {in }}$ & Oxygen inhibition constant & $m g l^{-1}$ & 0.001 & This study \\
\hline$\lambda$ & Biomass shear constant & $m m h^{-1}$ & 50 & This study \\
\hline$n$ & Exponent in the inhibition function & - & 20 & This study \\
\hline
\end{tabular}

Table 6. Initial-Boundary conditions for model Application 3.

\begin{tabular}{lccc}
\hline \hline Parameter & Symbol & Unit & Value \\
\hline COD concentration at $L=L(t)$ & $S_{1 L}$ & $\mathrm{mg} / L$ & 1000 \\
Oxygen concentration at $L=L(t)$ & $S_{2 L}$ & $\mathrm{mg} / L$ & 7 \\
Nitrate concentration at $L=L(t)$ & $S_{3 L}$ & $\mathrm{mg} / L$ & 400 \\
Nitrite concentration at $L=L(t)$ & $S_{4 L}$ & $\mathrm{mg} / L$ & 0 \\
NO concentration at $L=L(t)$ & $S_{5 L}$ & $\mathrm{mg} / L$ & 0 \\
$\psi$ concentration at $L=L(t)$ & $\psi_{L}$ & $\mathrm{mg} / L$ & 0 \\
Time Simulation & $\mathrm{T}$ & $\mathrm{h}$ & 552 \\
Initial Biofilm thickness & $L_{0}$ & $\mu m$ & 60 \\
Initial COD distribution & $S_{1,0}$ & $\mathrm{mg} / L$ & 0 \\
Initial Oxygen distribution & $S_{2,0}$ & $\mathrm{mg} / L$ & 0 \\
Initial Nitrate distribution & $S_{3,0}$ & $\mathrm{mg} / L$ & 0 \\
Initial Nitrite distribution & $S_{4,0}$ & $\mathrm{mg} / L$ & 0 \\
Initial NO distribution & $S_{5,0}$ & $\mathrm{mg} / L$ & 0 \\
\hline \hline
\end{tabular}



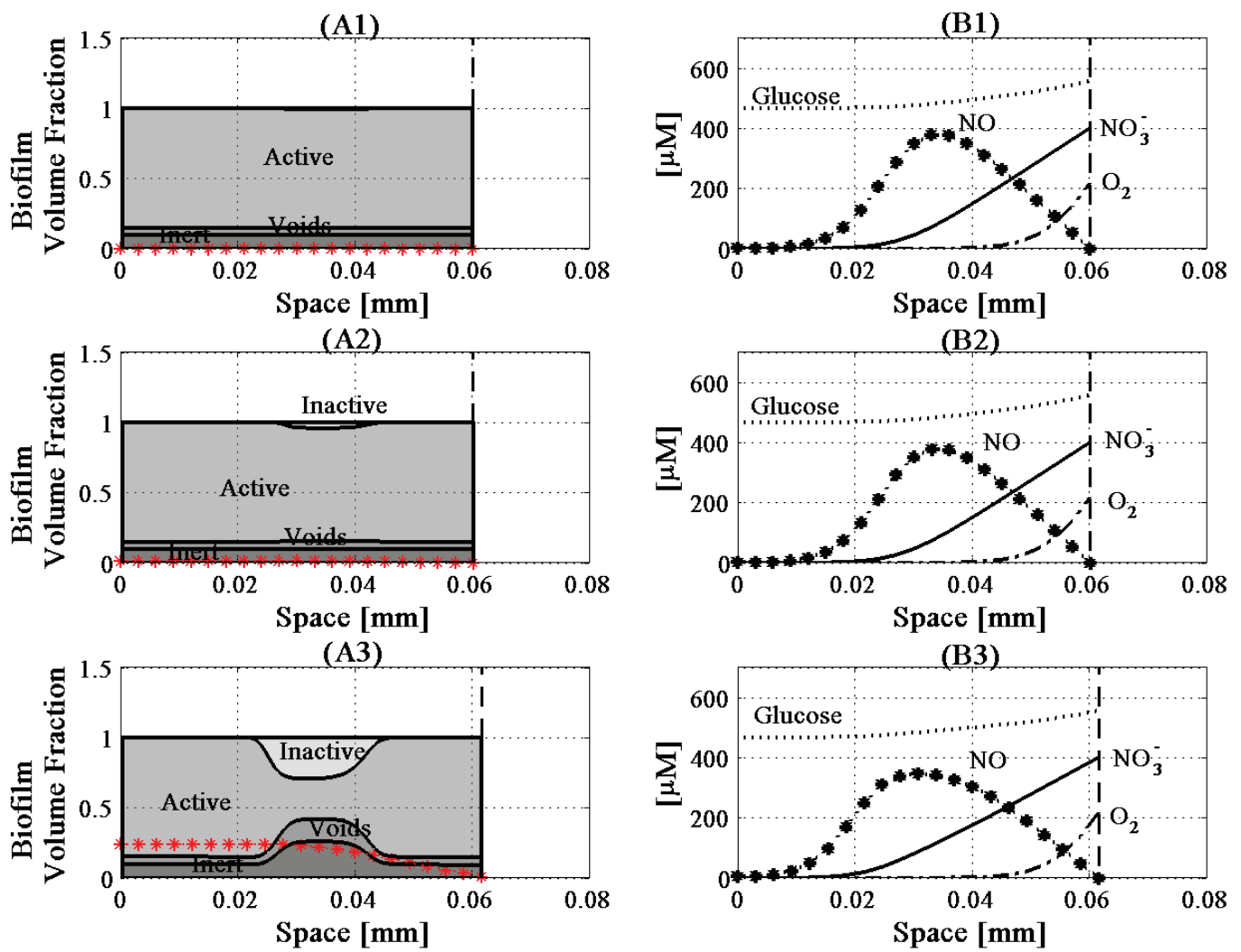

Figure 9. Effect of dispersal phenomenon induced by a self-produced biocide (NO) on bacterial volume fractions, $\psi_{1}$ profile (dotted-starred red line) and substrate concentration profiles in a batch system after 6 (A1,B1), 24 (A2,B2), 552 (A3,B3) hours (NO concentration is multiplied by 100 and Glucose is divided by 10). Simulation results refer to Application 3.

In the appendix 10, the model equations in matrix form are shown.

The dynamics of the described biofilm system are reported in figure (9) in terms of bacterial volume fractions and substrate profiles. Figure (9A1) shows biofilm composition after 6 hours simulation time: the biofilm is mostly constituted by active cells which perform both organic carbon oxidation and denitrification according to substrate distributions. In the central part of the biofilm, close to the transition zone, the active biomass start to convert to inactive due to the accumulation of $N O$ (fig9(B1)). Oxygen is consumed in the outmost part of the biofilm and the concentration drops to zero in the middle. Glucose is supplied in excess and hence fully penetrates. Nitrate concentration trend show a typical parabolic profile $[39,38]$ which reaches zero near the bottom of the biofilm.

After 24 hours simulation time the phenomenon of interest becomes evident: inactive biomass volume fraction increases and as is more apparent after 552 hours. Voids and inert fraction increase too (Figs 9(A3)). Continuing, inactive biomass fraction is predominant in the middle part of biofilm (9(A3)).

\section{Conclusion}

We have presented a mathematical model for mobility of free cells in biofilms induced by dispersal. Dispersal plays a crucial role in the survival of microbial species as it induces biofilm relocation on new surfaces upon unfavorable environmental conditions. In other cases biofilm dispersal represents the means of spread of pathogenic bacteria in human hosts. The study presents a theoretical model analyzing interaction between environmental cues triggering 
In the framework of continuous models, this study presents, to our knowledge, a first theoretical approach analyzing interaction between environmental cues triggering the cells release, including nutrient starvation, presence of toxic agent and self- produced biocide, and the dispersal phenomenon in multispecies biofilm. In particular the model considers the release of dispersal cells without neglecting the mechanical detachment due to the shear forces effect, in order to evaluate and predict the loss of biomass resulting from the two inherently different mechanisms.

The model is characterized by the tracing both the dynamics of microbial species growing in a sessile lifestyle and the motility of corresponding dispersed free cells originating from the inactivation of the biomass and further released into the bulk liquid. Those cells are modeled by introducing a new variable $\psi$, as firstly introduced in [24], in order to explicitly consider the different nature of sessile and dispersed cells. The release of dispersing cells is consequent to the environmental cues which are in turn explicitly modeled. The formation of voids due to the release of dispersed cells has been explicitly taken into account by modeling biofilm porosity as a state variable and introducing a reaction term which relates to the production of dispersed cells.

The complete model is a free boundary value problem for a system of nonlinear hyperbolic and parabolic partial differential equations. Qualitative properties of solutions showed the consistency of the model. Three illustrative biological cases for a PA biofilm have been studied by using numerical simulations developed by the method of characteristics. The model applications differ on the environmental cues that have been considered to trigger dispersal from biofilm: nutrient starvation in the first case, a non-lethal biofilm agent and a self-produced biocide in the second and third case respectively. The numerical results are in general agreement with experimental evidence and can particularly explain the hollowing of biofilm structure which is commonly observed when dispersal occurs. The model is able to predict dispersal occurrence in different locations of the biofilm. Indeed, in the first case, dispersal is shown to be initiated in the inner part of the biofilm with the inert concentration and biofilm porosity increasing over time. The second application is related to the release of dispersed cells from the outermost layers of the biofilm where the highest concentration of the inhibiting agent can be found. In the third case, the self-produced biocide is produced and temporary accumulates in the central part of the biofilm where it induces dispersal. Although the model applications are related to PA biofilms, the developed framework is general and could be applied to other biofilm systems as it captures many of the characteristics generally observed in biofilm dispersal.

For all the numerical studies performed in this work, the dispersal process has been considered as a continuous phenomenon as it is induced by continuous environmental cues. Future work might be related to simulation studies where the dispersal cues are considered instantaneous. In addition, a sensitivity analysis of the model parameters newly introduced in this work could be addressed in a future contribution. Based on the results achieved in [37, 40], existence and uniqueness of solutions to the mathematical problem can be proved by using the method of characteristics. Other relevant questions should be investigated, e. g. the generalization to problems regarding more complex biological cases and stability problems [41, 42].

\section{Acknowledgments}

The authors would like to acknowledge support from NSF/DMS 1517100, NIH Award No. R01GM109452 and the UNINA departmental grant 20152016 Analysis of Complex Biological Systems. 


\begin{tabular}{|c|c|c|c|c|c|c|c|c|c|c|}
\hline & Components & 1 & 2 & 3 & 4 & 5 & 6 & 7 & 8 & \\
\hline & Process & $S_{1}$ & $S_{2}$ & $X_{1}$ & $X_{2}$ & $X_{3}$ & $X_{4}$ & $X_{5}$ & $\psi_{1}$ & Process rate \\
\hline \multirow{6}{*}{$\begin{array}{l}1 \\
2 \\
3 \\
4 \\
5\end{array}$} & Growth of $X_{1}$ & $-\frac{1}{Y_{1}}$ & $-\frac{1}{Y_{2}}$ & $\left(1-K_{E P S}\right)$ & & & $K_{E P S}$ & & & $\mu_{1} X_{1}$ \\
\hline & Inactivation of $X_{1}$ & & & -1 & 1 & & & & & $\mu_{I 1} X_{1}$ \\
\hline & Decay of $X_{1}$ & & & -1 & & +1 & & & & $K_{d} X_{1}$ \\
\hline & Dispersal & & & & $-\left(\alpha_{1}+\alpha_{2}\right)$ & $\alpha_{1}$ & & $\alpha_{2}$ & $\alpha_{2}$ & $K_{1,2} X_{2}$ \\
\hline & Colonization & & & +1 & & & & & $-\frac{1}{Y_{c o l, 1}}$ & $r_{c o l, 1}$ \\
\hline & & $\frac{\Omega}{\tilde{0}}$ & 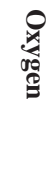 & 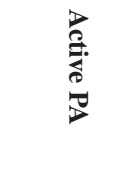 & 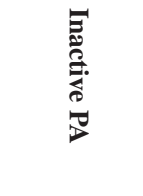 & $\stackrel{\Xi}{2}$ & 鲾 & & 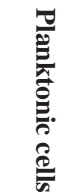 & \\
\hline
\end{tabular}

Table 7. Petersen matrix of the model Application 1.

Table 8. Kinetic rate expressions for model Application 1.

\begin{tabular}{lc}
\hline Process & Rate Expression \\
\hline 1. Growth of $X_{1}$ & $\mu_{1} X_{1}=\mu_{\max }\left(1-e^{-S_{1} / K s_{1}}\right)\left(1-e^{-S_{2} / K s_{2}}\right) X_{1}$ \\
2. Inactivation of $X_{1}$ & $\mu_{I 1} X_{1}=K_{I 1}\left(\frac{1}{1+S_{1} / I_{1}}\right) X_{1}$ \\
3. Decay of $X_{1}$ & $K_{d} X_{1}$ \\
4. Dispersal & $K_{i 2} X_{2}$ \\
5. Colonization & $r_{c o l, 1}=K_{c o l, 1} \frac{\psi_{1}}{k_{\psi, 1}+\psi_{1}}\left(1-e^{-S_{1} / K s_{1}}\right)\left(1-e^{-S_{2} / K s_{2}}\right)$ \\
\hline
\end{tabular}

\section{Appendix A}




\begin{tabular}{|c|c|c|c|c|c|c|c|c|c|c|c|}
\hline & Components & 1 & 2 & 3 & 4 & 5 & 6 & 7 & 8 & 9 & \\
\hline & Process & $S_{1}$ & $S_{2}$ & $S_{3}$ & $X_{1}$ & $X_{2}$ & $X_{3}$ & $X_{4}$ & $X_{5}$ & $\psi$ & Process rate \\
\hline \multirow{5}{*}{$\begin{array}{l}1 \\
2 \\
3 \\
4 \\
\end{array}$} & Growth of $X_{1}$ & $-\frac{1}{Y_{1}}$ & $-\frac{1}{Y_{2}}$ & & $\left(1-K_{E P S}\right)$ & & & $K_{E P S}$ & & & $\mu_{1} X_{1}$ \\
\hline & Inactivation of $X_{1}$ & & & $-\frac{1}{Y_{\theta}}$ & -1 & 1 & & & & & $\mu_{I 1} X_{1}$ \\
\hline & Decay of $X_{1}$ & & & & -1 & & +1 & & & & $K_{d} X_{1}$ \\
\hline & Dispersal & & & & & $-\left(\alpha_{1}+\alpha_{2}\right)$ & $\alpha_{1}$ & & $\alpha_{2}$ & $\alpha_{2}$ & $K_{i 2} X_{2}$ \\
\hline & & $\begin{array}{l}\Omega \\
\frac{\Omega}{0} \\
\frac{8}{0}\end{array}$ & 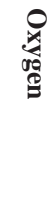 & 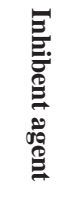 & $\begin{array}{l}3 \\
\frac{3}{3} \\
\frac{2}{2}\end{array}$ & 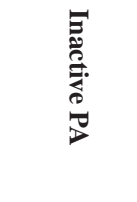 & $\underset{\frac{9}{2}}{\stackrel{5}{2}}$ & 国 & $\widetilde{\mathscr{B}}$ & 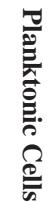 & \\
\hline
\end{tabular}

Table 9. Petersen matrix of the model Application 2.

Table 10. Kinetic rate expressions for model Application 2.

\begin{tabular}{lc}
\hline Process & Rate Expression \\
\hline 1. Growth of $X_{1}$ & $\mu_{1} X_{1}=\mu_{\max }\left(1-e^{-S_{1} / K s_{1}}\right)\left(1-e^{-S_{2} / K s_{2}}\right) X_{1}$ \\
2. Inactivation of $X_{1}$ & $\mu_{I 1} X_{1}=K_{I 1}\left(\frac{S_{3}}{K_{\theta}+S_{3}}\right) X_{1}$ \\
3. Decay of $X_{1}$ & $K_{d} X_{1}$ \\
4. Dispersal & $K_{i 2} X_{2}$ \\
\hline
\end{tabular}

\section{Appendix B}


10. Appendix C 


\begin{tabular}{|c|c|c|c|c|c|c|c|c|c|c|c|c|c|}
\hline & $\begin{array}{l}\text { Components } \\
\text { Process }\end{array}$ & $\begin{array}{ll}1 \\
S\end{array}$ & $\frac{2}{S}$ & $\begin{array}{l}3 \\
S_{3}\end{array}$ & $\begin{array}{ll}4 \\
\end{array}$ & \begin{tabular}{|l}
5 \\
$S_{4}$
\end{tabular} & $\frac{6}{X_{1}}$ & $\begin{array}{c}7 \\
X_{2}\end{array}$ & $\begin{array}{c}8 \\
X_{3}\end{array}$ & $\begin{array}{l}9 \\
X_{4}\end{array}$ & $\begin{array}{ll}10 \\
X_{5}\end{array}$ & 11 & Process rate \\
\hline \multirow{7}{*}{ 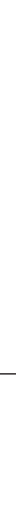 } & Aerobic & $-\frac{1}{-Y_{1}}$ & $\left(1-\frac{1}{y_{1}}\right)$ & & & & $\left(1-K_{E P S}\right)$ & & & $\frac{K_{E P S}}{K_{E P}}$ & & & $\mu_{1,1} X_{1}$ \\
\hline & $\begin{array}{l}\text { growth of } X_{1} \\
\text { Growth of } X_{1}\end{array}$ & $-\frac{1}{Y_{1}}$ & & $\left(1-\frac{1}{Y_{1}}\right) *\left(\frac{1}{1.14}\right)$ & $-\left(1-\frac{1}{Y_{1}}\right) *\left(\frac{1}{1.14}\right)$ & & $\left(1-K_{E P S}\right)$ & & & $K_{E P S}$ & & & $\mu_{1,2} X_{2}$ \\
\hline & Growth of $X_{1}$ & $-\frac{1}{Y_{1}}$ & & & $\left(1-\frac{1}{Y_{1}}\right) *\left(\frac{1}{0.58}\right)$ & $-\left(1-\frac{1}{Y_{1}}\right) *\left(\frac{1}{1.14}\right)$ & $\left(1-K_{E P S}\right)$ & & & $K_{E P S}$ & & & $\mu_{1,3} X_{3}$ \\
\hline & $\begin{array}{l}\text { Growth of } X_{1} \\
\text { on Nitric Ox- }\end{array}$ & $-\frac{1}{Y_{1}}$ & & & & $\left(1-\frac{1}{Y_{1}}\right) *\left(\frac{1}{0.58}\right)$ & $\left(1-K_{E P S}\right)$ & & & $K_{E P S}$ & & & $\mu_{1,4} X_{4}$ \\
\hline & $\begin{array}{l}\text { Inactivation } \\
\text { of } X\end{array}$ & & & & & & -1 & 1 & & & & & $\mu_{I 1} X_{1}$ \\
\hline & $\begin{array}{l}\text { Decay of } X_{1} \\
\text { Dispersal }\end{array}$ & & & & & & -1 & $-\left(\alpha_{1}+\alpha_{2}\right)$ & $\begin{array}{l}+1 \\
\alpha_{1}\end{array}$ & & $\alpha_{2}$ & $\alpha_{2}$ & $\begin{array}{l}K_{d} X_{1} \\
K_{i 2} X_{2}\end{array}$ \\
\hline & & $\begin{array}{l}\frac{2}{\overline{3}} \\
\overline{0}\end{array}$ & 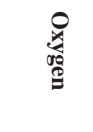 & $\frac{z}{\tilde{z}}$ & 晨 & 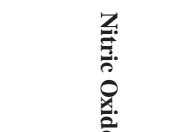 & $\begin{array}{l}\text { D. } \\
\text { ᄅ. } \\
0 \\
0\end{array}$ & 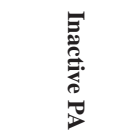 & 言 & 爵 & "ृ. & 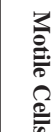 & \\
\hline
\end{tabular}

Table 11. Petersen matrix of the model Application 3. 
Table 12. Kinetic rate expressions for model Application 3.

\begin{tabular}{|c|c|}
\hline Process & Rate Expression \\
\hline 1. Aerobic growth of $X_{1}$ & $\mu_{1,1} X_{1}=\mu_{\max , 1} \frac{S_{1}}{K_{S_{1}}+S_{1}} \frac{S_{2}}{K_{S_{2}}+S_{2}} X_{1}$ \\
\hline 2. Growth of $X_{1}$ on Nitrate & $\mu_{1,2} X_{1}=\beta_{1} \mu_{\max , 1} \frac{S_{1}}{K_{S_{1}}+S_{1}} \frac{S_{3}}{K_{S_{3}}+S_{3}} \frac{K_{\text {in }}}{K_{\text {S }}+S_{2}} X_{1}$ \\
\hline 3. Growth of $X_{1}$ on Nitrite & 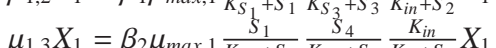 \\
\hline 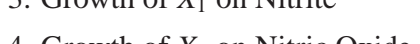 & $\mu_{1,3} \Lambda_{1}-\rho_{2} \mu_{\max , 1} \overline{K_{S_{1}}+S_{1}} \overline{K_{S_{4}}+S_{4}} \overline{K_{i n}+S_{2}} \Lambda_{1}$ \\
\hline 4. Growth of $X_{1}$ on Nitric Oxide & $\mu_{1,4} X_{1}=\beta_{3} \mu_{\max , 1} \frac{\mathrm{D}_{1}}{K_{S_{1}}+S_{1}} \frac{\mathrm{D}_{1}}{K_{S_{5}}+S_{5}} \frac{\Lambda_{i n}}{K_{\text {in }}+S_{2}} X_{1}$ \\
\hline 5. Inactivation of $X_{1}$ & $\mu_{I 1} X_{1}=K_{I 1}\left(\frac{S_{5}^{n}}{K_{N O}^{n}+S_{5}^{n}}\right) X_{1}$ \\
\hline 6. Decay of $X_{1}$ & $K_{d} X_{1}$ \\
\hline 7. Dispersal & $K_{i 2} X_{2}$ \\
\hline
\end{tabular}

\section{References}

[1] D. Schleheck, N. Barraud, J. Klebensberger, J. S. Webb, D. McDougald, S. A. Rice, S. Kjelleberg, Pseudomonas aeruginosa pao1 preferentially grows as aggregates in liquid batch cultures and disperses upon starvation, PloS one 4 (5) (2009) e5513.

[2] H.-C. Flemming, J. Wingender, The biofilm matrix, Nature Reviews Microbiology 8 (9) (2010) 623-633.

[3] I. Sutherland, The biofilm matrix-an immobilized but dynamic microbial environment, Trends in microbiology 9 (2001) $222-227$.

[4] T. Tolker-Nielsen, U. C. Brinch, P. C. Ragas, J. B. Andersen, C. S. Jacobsen, S. Molin, Development and dynamics of pseudomonassp. biofilms, Journal of Bacteriology 182 (22) (2000) 6482-6489.

[5] F. Villa, S. Villa, A. Gelain, F. Cappitelli, Sub-lethal activity of small molecules from natural sources and their synthetic derivatives against biofilm forming nosocomial pathogens, Current topics in medicinal chemistry 13 (24) (2013) 3184-3204.

[6] S. Kjelleberg, M. Givskov, The biofilm mode of life: mechanisms and adaptations, Horizon Scientific Press, 2007.

[7] D. McDougald, S. A. Rice, N. Barraud, P. D. Steinberg, S. Kjelleberg, Should we stay or should we go: mechanisms and ecological consequences for biofilm dispersal, Nature Reviews Microbiology 10 (1) (2012) 39-50.

[8] P. Stoodley, K. Sauer, D. Davies, J. W. Costerton, Biofilms as complex differentiated communities, Annual Reviews in Microbiology 56 (1) (2002) 187-209.

[9] F. Villa, F. Cappitelli, Plant-derived bioactive compounds at sub-lethal concentrations: towards smart biocide-free antibiofilm strategies, Phytochemistry reviews 12 (1) (2013) 245-254.

[10] S. M. Hunt, E. M. Werner, B. Huang, M. A. Hamilton, P. S. Stewart, Hypothesis for the role of nutrient starvation in biofilm detachment, Applied and environmental microbiology 70 (12) (2004) 7418-7425.

[11] J. D. Chambless, P. S. Stewart, A three-dimensional computer model analysis of three hypothetical biofilm detachment mechanisms, Biotechnology and bioengineering 97 (6) (2007) 1573-1584.

[12] S. M. Hunt, M. A. Hamilton, J. T. Sears, G. Harkin, J. Reno, A computer investigation of chemically mediated detachment in bacterial biofilms, Microbiology 149 (5) (2003) 1155-1163.

[13] J. Dockery, I. Klapper, Finger formation in biofilm layers, SIAM J. Appl. Math. 62 (2002) 853-869.

[14] E. Alpkvist, I. Klapper, A multidimensional multispecies continuum model for heterogeneous biofilm development, Bull Math Biol 69 (2007) 765-789.

[15] I. Klapper, J. Dockery, Mathematical description of microbial biofilms, SIAM Rev. 52 (2010) 221-265.

[16] J. B. Xavier, C. Picioreanu, S. A. Rani, M. C. van Loosdrecht, P. S. Stewart, Biofilm-control strategies based on enzymic disruption of the extracellular polymeric substance matrix-a modelling study, Microbiology 151 (12) (2005) 3817-3832.

[17] B. O. Emerenini, B. A. Hense, C. Kuttler, H. J. Eberl, A mathematical model of quorum sensing induced biofilm detachment, PloS one 10 (7) (2015) e0132385

[18] B. Emerenini, S. Sonner, H. Eberl, Mathematical analysis of a quorum sensing induced biofilm dispersal model and numerical simulation of hollowing effects., Mathematical biosciences and engineering: MBE 14 (3) (2017) 625.

[19] B. Atkinson, I. Davies, The overall rate of substrate uptake (reaction) by microbial films, Trans. Inst. Chem. Engrs. 52 (1974) $248-259$.

[20] J. Kissel, P. McCarty, R. Street, Numerical simulations of mixed-culture biofilms, J Environ Eng 110 (1984) 393-411.

[21] O. Wanner, W. Gujer, A multispecies biofilm model, Biotechnol Bioeng 28 (1986) 314-328.

[22] O. Wanner, P. Reichert, Mathematical modelling of mixed-culture biofilms, Biotechnol Bioeng 49 (1996) $172-184$.

[23] B. D'Acunto, L. Frunzo, Qualitative analysis and simulations of a free boundary problem for mulispecies biofilm models, Math Comput Model 53 (2011) 1596-1606.

[24] B. DAcunto, L. Frunzo, I. Klapper, M. Mattei, Modeling multispecies biofilms including new bacterial species invasion, Mathematical biosciences 259 (2015) 20-26.

[25] A. Ito, T. May, K. Kawata, S. Okabe, Significance of rpos during maturation of escherichia coli biofilms, Biotechnology and bioengineering 99 (6) (2008) 1462-1471.

[26] M. Hentzer, L. Eberl, M. Givskov, Transcriptome analysis of pseudomonas aeruginosa biofilm development: anaerobic respiration and iron limitation, Biofilms 2 (01) (2005) 37-61.

[27] K. Sauer, M. Cullen, A. Rickard, L. Zeef, D. Davies, P. Gilbert, Characterization of nutrient-induced dispersion in pseudomonas aeruginosa pao1 biofilm, Journal of bacteriology 186 (21) (2004) 7312-7326. 
[28] M. Gjermansen, P. Ragas, C. Sternberg, S. Molin, T. Tolker-Nielsen, Characterization of starvation-induced dispersion in pseudomonas putida biofilms, Environmental microbiology 7 (6) (2005) 894-904.

[29] N. Barraud, D. Schleheck, J. Klebensberger, J. S. Webb, D. J. Hassett, S. A. Rice, S. Kjelleberg, Nitric oxide signaling in pseudomonas aeruginosa biofilms mediates phosphodiesterase activity, decreased cyclic di-gmp levels, and enhanced dispersal, Journal of bacteriology 191 (23) (2009) 7333-7342.

[30] N. Barraud, M. V. Storey, Z. P. Moore, J. S. Webb, S. A. Rice, S. Kjelleberg, Nitric oxide-mediated dispersal in single-and multi-species biofilms of clinically and industrially relevant microorganisms, Microbial biotechnology 2 (3) (2009) 370-378.

[31] B. DAcunto, G. Esposito, L. Frunzo, M. Mattei, F. Pirozzi, Mathematical modeling of heavy metal biosorption in multispecies biofilms, Journal of Environmental Engineering (2015) C4015020.

[32] B. V. Merkey, B. E. Rittmann, D. L. Chopp, Modeling how soluble microbial products (smp) support heterotrophic bacteria in autotroph-based biofilms, Journal of theoretical biology 259 (4) (2009) 670-683.

[33] B. Purevdorj-Gage, W. Costerton, P. Stoodley, Phenotypic differentiation and seeding dispersal in non-mucoid and mucoid pseudomonas aeruginosa biofilms, Microbiology 151 (5) (2005) 1569-1576.

[34] B. D’Acunto, G. Esposito, L. Frunzo, F. Pirozzi, Dynamic modeling of sulfate reducing biofilms, Comput Math Appl 62 (2011) $2601-2608$.

[35] B. D'Acunto, L. Frunzo, Free boundary problem for an initial cell layer in multispecies biofilm formation, Appl Math Lett 25 (2012) $20-26$.

[36] H. Beyenal, S. N. Chen, Z. Lewandowski, The double substrate growth kinetics of pseudomonas aeruginosa, Enzyme and Microbial Technology 32 (1) (2003) 92-98.

[37] B. D'Acunto, L. Frunzo, M. Mattei, Qualitative analysis of the moving boundary problem for a biofilm reactor model, Journal of Mathematical Analysis and Applications 438 (1) (2016) 474-491.

[38] M. Mattei, L. Frunzo, B. DAcunto, G. Esposito, F. Pirozzi, Modelling microbial population dynamics in multispecies biofilms including anammox bacteria, Ecological Modelling 304 (2015) 44-58.

[39] J. Holman, D. Wareham, Cod, ammonia and dissolved oxygen time profiles in the simultaneous nitrification/denitrification process, Biochemical Engineering Journal 22 (2) (2005) 125-133.

[40] L. Frunzo, M. Mattei, Qualitative analysis of the invasion free boundary problem in biofilms, Ricerche di Matematica 66 (1) (2017) 171-188.

[41] F. Capone, R. De Luca, S. Rionero, On the stability of non-autonomous perturbed lotka-volterra models, Applied Mathematics and Computation 219 (12) (2013) 6868-6881.

[42] F. Capone, V. De Cataldis, R. De Luca, On the nonlinear stability of an epidemic seir reaction-diffusion model, Ricerche di Matematica 62 (1) (2013) 161-181. 